\title{
COHOMOLOGY OF LOCAL GROUP EXTENSIONS $\left({ }^{1}\right)$
}

\author{
S. ŚWIERCZKOWSKI
}

1. Introduction. A local group is an object which, roughly speaking, satisfies the usual axioms of a group, except that the product $x y$ may not be defined for some $x, y$; the existence of identity and inverses are assumed. An example is a subset of a group which contains the identity and with every $x$ also its inverse $x^{-1}$. Such a local group will be called enlargeable. Suppose $U$ is a local group containing a group $N$ such that all products of the form $u n, n u, u n u^{-1}(u \in U, n \in N)$ are defined and $u n u^{-1} \in N(N$ is a normal subgroup of $U)$. Then the quotient space $V=U / N$ of the cosets of $N$ is a local group in an obvious way. We shall consider here the problem of enlarging the above local group $U$ under the assumption that $V$ is enlargeable. This question has been considered previously by W. T. van Est [5] and P. A. Smith ([13], [14]). The present paper originated in the attempt to derive the results of [14] by the homological methods of [5]; it is therefore in the same spirit as [5], where method is concerned, and among our results are the main theorems of [14].

A rough summary is as follows. In $\S 2$ we introduce some basic notions. We say that

$$
\mathfrak{L}: 1 \longrightarrow N \subset U \underset{\phi}{\longrightarrow} V \longrightarrow 1
$$

is a local extension over $V$ if $N$ is a normal subgroup of $U, V$ is isomorphic to $U / N$ and $\phi$ is canonical. We call $\mathfrak{L}$ enlargeable over a group $G$ if $V \subset G$ and there exists a group $H$ containing $U$ and a homomorphism $\phi^{\prime}: H \rightarrow G$ such that $\phi^{\prime} \mid U=\phi$. Then the exact sequence of groups $1 \rightarrow N \subset H \rightarrow G_{\phi^{\prime}} \rightarrow 1$ is called an enlargement over $G$ of $\mathfrak{R}$. It is easily observed that $\mathfrak{L}$ is enlargeable (over some group) iff $U$ is enlargeable, and henceforth only the problem of enlarging $\mathfrak{L}$ over a fixed group $G$ is considered.

$\S \S 3,4$ and 5 introduce the basic technical apparatus: complexes and cohomology groups associated with a local group $V$ and a group $G$ containing it. We begin by introducing in $\S 3$ the simplicial scheme $\Gamma_{G}^{V}$ (first considered in [14]) the set of whose vertices is $G$ and whose simplexes are those sets $\left\{g_{0}, \ldots, g_{n}\right\}$ which satisfy $g_{i}^{-1} g_{j} \in V$ for all $i, j$. This scheme is connected and simply connected iff $G$ is $V$-monodrome (that is when $V$ generates $G$ and any other group $K$ generated by $V$ is a homomorphic image of $G$, the homomorphism being on $V$ the identity). We investigate more closely the role of the first homotopy group of $\Gamma_{G}^{V}$ in the problem of enlarging

Received by the editors August 25, 1966.

( $\left.{ }^{1}\right)$ Supported in part by National Science Foundation Grant GP2439. 
a homomorphism $V \rightarrow H$ ( $H$ a group) to $G \rightarrow H$, and we define an obstruction to such enlargement.

In $\S 4$ we consider the complex $\subseteq\left(\Gamma_{G}^{V}\right)$ of singular chains of $\Gamma_{G}^{V}$. This is a $G$ complex (i.e. a complex of $G$-modules whose boundary operators are $G$-morphisms). In particular $\mathbb{E}\left(\Gamma_{G}^{G}\right)$ is the standard homogeneous $Z G$-resolution of $Z$, so for any $G$-module $C$ we have $H^{n}(G, C)=H_{\mathrm{eq}}^{n}\left(\mathbb{E}\left(\Gamma_{G}^{G}\right), C\right)$, where "eq" means that this is the cohomology of the complex $\operatorname{Hom}_{G}\left(\mathbb{S}\left(\Gamma_{G}^{G}\right), C\right.$ ) (and not $\operatorname{Hom}_{z}\left(\mathbb{S}\left(\Gamma_{G}^{G}\right), C\right)$ ). We define by analogy $H^{n}(V, C)=H_{\mathrm{eq}}^{n}\left(\mathfrak{\subseteq}\left(\Gamma_{G}^{V}\right), C\right)$, and

$$
H^{n}(G \bmod V, C)=H_{\mathrm{eq}}^{n}\left(\mathfrak{S}\left(\Gamma_{G}^{G}\right) \bmod \Subset\left(\Gamma_{G}^{V}\right), C\right) .
$$

Expressing these groups in terms of the standard nonhomogeneous $Z G$-resolution of $Z$, one finds that $H^{n}(V, C)$ does not depend on $G$, nor on the assumption that $V$ is enlargeable, so it can be redefined for an arbitrary local group $V$ and a $V$-module $C$.

$\S \S 5$ and 6 establish a fundamental isomorphism

$$
\kappa: H^{n}(G \bmod V, C) \rightarrow \operatorname{Hom}_{G}\left(H_{n-1}\left(\Gamma_{G}^{V}\right), C\right)(n \geqq 2)
$$

under the assumption that $\Gamma_{G}^{V}$ is connected and acyclic in dimensions $1, \ldots, n-2$.

$\$ 7$ introduces the link between the above and local extensions. We describe the latter in terms of multiplicators (analogous to the factor sets in the theory of O. Schreier, [17, p. 89]) and in slightly more detail than this is done in [14]. A homomorphism $\Theta: V \rightarrow A(N) / I(N)$ of the local group $V$ into the automorphism group of $N$ modulo the subgroup of inner automorphisms is called an abstract kernel and denoted by $\langle V, N, \Theta\rangle$. With a local extension $\mathfrak{Q}$ (see (1)) we associate the abstract kernel

$$
\Theta: V \underset{\psi}{\longrightarrow} \underset{\iota_{N}}{\longrightarrow} A(N) \longrightarrow A(N) / I(N)
$$

where $\psi$ is any map which composed with $\phi$ is the identity, $\iota_{N}$ assigns to every $u \in U$ the automorphism $n \rightarrow u n u^{-1}$, and the last map is canonical. With any abstract kernel $\langle V, N, \Theta\rangle$ we associate an obstruction $\operatorname{Obs}\langle V, N, \Theta\rangle \in H^{3}(V, C)$ ( $C=$ center $N$ with $V$-structure defined by the kernel) which vanishes iff $\langle V, N, \Theta\rangle$ is the abstract kernel (2) of some local extension $\mathfrak{Q}$. This discussion is very much modelled on that by S. Eilenberg and S. MacLane in [3].

Also by analogy with [3] we define in $\S 8$ for any abstract kernel $\langle G, N, \Theta\rangle$ whose restriction $\langle V, N, \Theta \mid V\rangle$ is of the form (2), for some local extension $\mathbb{Q}$, an obstruction $\operatorname{Obs}_{\mathfrak{R}}\langle G, N, \Theta\rangle \in H^{3}(G \bmod V, C)$ which vanishes iff $\langle G, N, \Theta\rangle$ is the abstract kernel of some enlargement over $G$ of $\mathfrak{Q}$.

In $\S 9$ we interpret $H^{2}(V, C)$ as the set of isomorphism classes of local extensions having a common abstract kernel $\langle V, N, \Theta\rangle$ which induces on $C$ the given $V$ module structure. Similarly $H^{2}(G \bmod V, C)$ is interpreted as the set of isomorphism classes of enlargements over $G$ of $\mathfrak{Q}$ which have a common abstract kernel $\langle G, N, \Theta\rangle$. The analogy with [3] is maintained. 
The main results about the enlargeability over $G$ of $\mathfrak{L}$, where $V$ generates $G$, are given in $\$ 10$. Trying to find an enlargement over $G$ of $\mathfrak{L}$ we first attempt to enlarge the abstract kernel (2) of $\mathfrak{Q}$; we meet an obstruction of the kind described in $\S 3$. If this vanishes (it always does when $G$ is $V$-monodrome), we have a unique enlargement $\langle G, N, \Theta\rangle$ of the abstract kernel of $\mathfrak{E}$, and an enlargement over $G$ of $\mathfrak{L}$ (if it exists) must have the kernel $\langle G, N, \Theta\rangle$. Thus, by the results of $\S 8, \mathfrak{Q}$ is enlargeable over $G$ iff $\operatorname{Obs}_{\mathfrak{E}}\langle G, N, \Theta\rangle$ vanishes. Applying this also to a subextension $\mathfrak{L}^{\wedge}$ of $\mathfrak{L}$ (i.e. $N \subset \hat{U} \subset U, \hat{V}=\hat{U} / N$ ) we recover the fundamental theorem of P. A. Smith [14, (50.7), p. 423], in a slightly more general form. In case when $N$ is abelian, the enlargeability of $\mathfrak{Q}$ was discussed in ([5], [6]) and the second obstruction was found to be a 2-dimensional cohomology class. We give a new proof of this result.

In $\S 11$ it is assumed that $G$ is a topological group with $H_{2}^{\text {top }}(G)=0$ and $V$ is open in $G$. A simple sufficient condition for the enlargeability over $G$ of a subextension $\mathfrak{L}^{\wedge}$ is deduced from the theorems of $\S 10$. Related results can be found in [13] (without proof) and in [5].

In $\$ 12$ we give a theorem (announced in [15]) concerning the enlargeability of infinite dimensional local Lie groups. When applied to the finite dimensional case, it gives the enlargeability of a local Lie group, as a corollary of the known fact that the second homotopy of a Lie group vanishes.

I would like to express my warmest thanks to W. T. van Est who at some time has carried out investigations similar to those outlined above, and who most kindly made available to me his unpublished notes. Most basic ideas and constructions below can be traced back to these. Subsequent correspondence with him resulted in many improvements of my original presentation, for this also I am very indebted.

2. Local groups and extensions. A local group is a set $V$ such that for certain pairs $\langle x, y\rangle$ in $V \times V$ there exists an element $x y$ in $V$, called the product of $x$ by $y$ and satisfying the conditions

(i) if $x y$ and $y z$ exist, then $(x y) z$ and $x(y z)$ exist or do not exist simultaneously, and $(x y) z=x(y z)$ if they do exist,

(ii) there is a unit 1 in $V$ such that $1 x=x 1=x$ for every $x$,

(iii) every $x$ in $V$ has a unique inverse $x^{-1}$ satisfying $\left(x^{-1}\right)^{-1}=x$ and $x x^{-1}=1$,

(iv) if $x y$ exists, then $y^{-1} x^{-1}$ exists (it follows from the above that this is $(x y)^{-1}$ ). A map of local groups $\phi: V \rightarrow V^{\prime}$ will be called a morphism if the existence of $x y$ in $V$ implies that $\phi(x) \phi(y)$ exists in $V^{\prime}$ and equals $\phi(x y)$. With these morphisms local groups form a category which contains the subcategory of groups. Henceforth the word "morphism" will be reserved to mean a morphism in any of these categories; in the category of sets we shall use the word map. A morphism $\phi: V \rightarrow V^{\prime}$ will be called strong if, for every $x, y$ in $V$, the existence of $x y$ is equivalent to the existence of $\phi(x) \phi(y)$. A morphism will be called a mono- or epimorphism if, considered as a map, it is injective or surjective. 
A subset of a local group will be called symmetric if it contains the identity and if it contains with every element $x$ also its inverse $x^{-1}$. A symmetric subset of a local group is again a local group. Since a morphism $\phi: V \rightarrow V^{\prime}$ preserves the identity and inverses, the sets $\phi V$ and $\phi^{-1} 1$ are local groups; we call them the image and kernel of $\phi$. A sequence

$$
\cdots \longrightarrow V^{\prime} \underset{\phi_{i}}{\longrightarrow} V_{\phi_{i+1}} V^{\prime \prime} \longrightarrow \cdots
$$

is called exact if $\operatorname{Im} \phi_{i}=\operatorname{Ker} \phi_{i+1}$ for every $i$.

By a local extension $\mathfrak{Q}$ over $V$ we shall mean an exact sequence of local groups

$$
\mathfrak{L}: 1 \longrightarrow N \subset U \underset{\phi}{\longrightarrow} V \longrightarrow 1,
$$

where the morphism $\phi$ is strong. This implies that $N$ is a group. It also follows that $u n, n u$ and $u n u^{-1}$ exist for every $u \in U, n \in N$, and $u n u^{-1} \in N$. If also $U, V$ are groups, then we shall call $\mathfrak{Q}$ a global extension.

We shall say that a local group $V$ is enlargeable if there exists a group $G$ and a morphism $\phi: V \rightarrow G$ such that $\phi: V \rightarrow \phi V$ is an isomorphism. If, in particular $V \subset G$ then we shall say that $G$ is $V$-monodrome provided that

(*) $V$ generates $G$ and for every morphism $\phi: V \rightarrow H$, where $H$ is a group, there exists a morphism $\nu: G \rightarrow H$ which makes the diagram

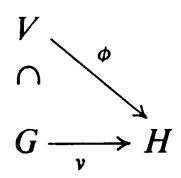

commutative.

If $V$ is enlargeable, there always exists a $V$-monodrome group; it is enough to take the group presented by the set $V$, as set of generators, with the set of relations equal to the multiplication table of the local group $V[15$, p. 222].

By a morphism $\mathfrak{L} \rightarrow \mathfrak{L}^{\prime}$ of local extensions we shall mean a commutative diagram

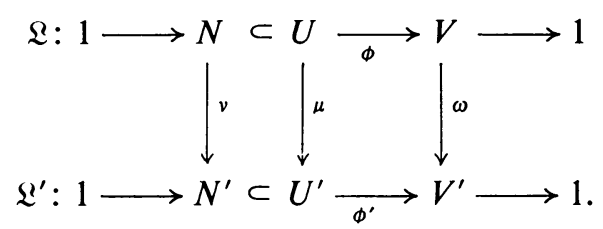

If, in particular, $N^{\prime}=N, U^{\prime}$ and $V^{\prime}$ are groups, $\nu$ is the identity and $\mu, \omega$ are inclusions, then $\mathfrak{L}$ will be called enlargeable to the global extension $\mathfrak{L}^{\prime}$, and $\mathfrak{L}^{\prime}$ will be called an enlargement over $V^{\prime}$ of $\mathfrak{L}$. We shall also say in this case that $\mathfrak{L}$ is enlargeable over $V^{\prime}$, or just enlargeable.

It is easy to see that the problem of enlarging a local group $U$ which appears as the middle term of some local extension $\mathfrak{Q}$ is equivalent to the problem of enlarging $\mathfrak{L}$. Indeed, if $U$ is enlargeable, we can take a group $U^{\prime}$ generated by $U$ and take $V^{\prime}=U^{\prime} \mid N$. If, in particular we take $U^{\prime}$ to be $U$-monodrome, then, as can be shown 
rather easily, $V^{\prime}=U^{\prime} / N$ will be $V$-monodrome. This shows that $\mathfrak{L}$ is enlargeable over some group containing $V$ iff $\mathfrak{L}$ is enlargeable over a $V$-monodrome group.

If $\mathbb{B S}_{i}: 1 \rightarrow N \subset H_{i} \rightarrow G \rightarrow 1(i=0,1)$ are two enlargements of $\mathfrak{Q}$, then by a morphism $\mathscr{S}_{0} \rightarrow \mathscr{S}_{1}$ over $\mathfrak{Q}$ we shall mean a commutative diagram

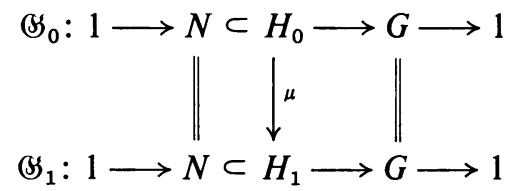

where $\mu \mid U=$ identity.

3. The simplicial scheme $\Gamma_{G}^{V}$. We shall associate with a group $G$ and a symmetric $V \subset G$ the simplicial scheme $\Gamma_{G}^{V}$, the set of whose vertices is $G$, such that $\left\{g_{0}, g_{1}, \ldots, g_{n}\right\} \subset G$ is a simplex iff $g_{i}^{-1} g_{j} \in V$ for all $i, j$. Let $\pi_{1}\left(\Gamma_{G}^{V}\right)$ denote the first homotopy group of $\Gamma_{G}^{V}$.

LEMMA 1. If $V$ generates $G$, then for every morphism $h_{V}: V \rightarrow H$, where $H$ is a group, there exists a morphism

$$
\rho\left(h_{V}\right): \pi_{1}\left(\Gamma_{G}^{V}\right) \rightarrow H
$$

which is trivial if and only if $h_{V}$ can be enlarged to a morphism $h: G \rightarrow H$.

DEFINITION. $\rho\left(h_{V}\right)$ will be called the obstruction to the enlargeability of $h_{V}$ over $G$, and denoted by $\operatorname{Obs}\left(h_{V}, G\right)$. Instead of saying that the morphism $\operatorname{Obs}\left(h_{V}, G\right)$ is trivial, we shall say that it vanishes.

To define $\rho\left(h_{V}\right)$, we take a loop $l$ at 1 in $\Gamma_{G}^{V}$ and write it as $l=\left\langle g_{0}, g_{1}, \ldots, g_{n}\right\rangle$, where $g_{0}=g_{n}=1$ and $g_{i-1}^{-1} g_{i}=v_{i} \in V(i=1, \ldots, n)$. We define $\rho\left(h_{V}\right) l=h_{V}\left(v_{1}\right) h_{V}\left(v_{2}\right) \ldots$ $h_{v}\left(v_{n}\right)$. A loop $l^{\prime}$ will be said to arise from $l$ by an elementary deformation if $l^{\prime}$ is of one of the forms

$$
\left\langle g_{0}, \ldots, g_{i}, g_{i+2}, \ldots, g_{n}\right\rangle \text { or }\left\langle g_{0}, \ldots, g_{i}, g, g_{i+1}, \ldots, g_{n}\right\rangle,
$$

where $g_{i}^{-1} g_{i+2} \in V$ in the first case and $g_{i}^{-1} g, g^{-1} g_{i+1} \in V$ in the second. Since $h_{V}$ is a morphism, it is easy to see that then $\rho\left(h_{V}\right) l^{\prime}=\rho\left(h_{V}\right) l$. A homotopy between two loops can be always accomplished by a sequence of elementary deformations, hence $\rho\left(h_{V}\right) l$ depends only on the homotopy class $l$ of $l$. We define now (3.1) by putting $\rho\left(h_{V}\right) \bar{l}=\rho\left(h_{V}\right) l$. It is clear that the map so obtained is a morphism.

Suppose (3.1) is trivial. To define $h: G \rightarrow H$, take any $g \in G$, write $g$ as a product $v_{1} v_{2} \cdots v_{n}$ of elements of $V$ and put

$$
h(g)=h_{V}\left(v_{1}\right) \cdot h_{v}\left(v_{2}\right) \cdots h_{v}\left(v_{n}\right) .
$$

To show that $h(g)$ does not depend on the presentation of $g$, assume $g=v_{1}^{\prime} v_{2}^{\prime} \cdots v_{m}^{\prime}$ $\left(v_{i}^{\prime} \in V\right)$ and define a loop $l=\left\langle g_{0}, \ldots, g_{n}, g_{m}^{\prime}, g_{m-1}^{\prime}, \ldots, g_{0}^{\prime}\right\rangle$ by

$$
g_{0}=g_{0}^{\prime}=1, \quad g_{k}=v_{1} \cdots v_{k} \quad(1 \leqq k \leqq n), \quad g_{k}^{\prime}=v_{1}^{\prime} \cdots v_{k}^{\prime} \quad(1 \leqq k \leqq m) .
$$


Since $\rho\left(h_{V}\right) l=1$, we obtain that the right-hand side of (3.2) is equal to $h_{V}\left(v_{1}^{\prime}\right) h_{V}\left(v_{2}^{\prime}\right) \cdots$ $h_{V}\left(v_{m}^{\prime}\right)$ which proves the assertion. Obviously $h$ is an enlargement of $h_{V}$.

Conversely, assuming that $h_{V}$ is enlargeable to $h: G \rightarrow H$, we have for $l=\left\langle g_{0}, \ldots, g_{n}\right\rangle$ as above, $\rho\left(h_{V}\right) l=h\left(v_{1}\right) h\left(v_{2}\right) \cdots h\left(v_{n}\right)=h\left(v_{1} \cdots v_{n}\right)=h(1)=1$, whence $\rho\left(h_{V}\right)$ is trivial.

LEMMA 2. $G$ is V-monodrome iff $\Gamma_{G}^{V}$ is 1-connected (i.e. connected and simply connected).

This is proved in [14, (35.4), p. 399]. We shall give a slightly different proof based on the preceding lemma.

Proof. Suppose $\Gamma_{G}^{V}$ is 1-connected. The connectedness implies that $V$ generates $G$. The simple connectedness means that $\pi_{1}\left(\Gamma_{G}^{V}\right)=1$ whence, by the previous lemma, every morphism of $V$ into a group $H$ can be enlarged to a morphism $G \rightarrow H$. Thus $G$ is $V$-monodrome.

Now suppose $G$ is $V$-monodrome. Then $\Gamma_{G}^{V}$ is connected because $V$ generates $G$. Let $\tilde{\Gamma}_{G}^{V}$ be the 1-connected covering scheme, so that the vertices of $\tilde{\Gamma}_{G}^{V}$ are equivalence classes of paths in $\Gamma_{G}^{V}$ originating from 1, the equivalence being homotopy with fixed end-point (for a precise definition see [14, (10.2), p. 378]). Let $\widetilde{G}$ be the set of vertices of $\tilde{\Gamma}_{G}^{v}$, we make $\tilde{G}$ into a group by path composition, i.e. if $a, b$ are paths representing $\tilde{a}, \tilde{b} \in \tilde{G}$, then the path $a$ followed by $g b$, where $g$ is the endpoint of $a$, represents $\tilde{a} \tilde{b}$. $V$ is contained in $\tilde{G}$ (identifying $v \in V$ with the class of the path $\langle 1, v\rangle$ ) as a set of generators, and the operation of assigning to a path its end-point induces a morphism $\tilde{G} \rightarrow G$ which is on $V$ the identity. Its kernel is $\pi_{1}\left(\Gamma_{G}^{V}\right)$. But since $G$ is $V$-monodrome, there is a morphism $G \rightarrow \tilde{G}$ equal to the identity on $V$, so that the two morphisms between $G$ and $\tilde{G}$ are inverse to each other (the groups being generated by $V$ ), whence $\pi_{1}\left(\Gamma_{G}^{V}\right)=1$.

COROllary. If $G$ is $V$-monodrome, then $H_{0}\left(\Gamma_{G}^{V}\right)=Z$ and $H_{1}\left(\Gamma_{G}^{V}\right)=0$.

We consider now another group $\hat{G}$, a symmetric $\hat{V} \subset \hat{G}$, and a morphism

$$
\omega:(\hat{V}, \hat{G}) \rightarrow(V, G) ;
$$

(this means a morphism $\omega: \hat{G} \rightarrow G$ such that $\omega \hat{V} \subset V$ ). The induced map of schemes $\Gamma_{G \wedge}^{V \wedge} \rightarrow \Gamma_{G}^{V}$ gives a morphism $\omega_{\pi}: \pi_{1}\left(\Gamma_{G \wedge}^{V \wedge}\right) \rightarrow \pi_{1}\left(\Gamma_{G}^{V}\right)$.

LEMmA 3. If $V$ generates $G, \hat{V}$ generates $\hat{G}$ and $h_{V}: V \rightarrow H$ is a morphism into a group $H$, then

$$
\operatorname{Obs}\left(h_{V} \circ \omega, \hat{G}\right)=\operatorname{Obs}\left(h_{V}, G\right) \circ \omega_{\pi} .
$$

Indeed, denoting $h_{\hat{V}}=h_{V} \circ \omega, \hat{\rho}\left(h_{\hat{V}}\right)=\operatorname{Obs}\left(h_{\hat{V}}, \hat{G}\right)$ we have from the proof of Lemma 1

$$
\hat{\rho}\left(h_{\hat{V}}\right) l=\rho\left(h_{V}\right)(\omega l)
$$

for any loop $l$ at 1 in $\Gamma_{G \wedge}^{V \wedge}$. It remains to pass to homotopy classes. 
4. Chain complexes associated with $\Gamma_{G}^{V}$. A chain or cochain complex $X$ of abelian groups $X_{n}$ will be called a $G$-complex if each $X_{n}$ is a $G$-module (left module over the group-ring $Z G)$ and the differential $d: X_{n} \rightarrow X_{n+r}(r= \pm 1)$ is a $G$ morphism (morphism in the category of $G$-modules). If $X$ is a chain $G$-complex and $C$ is a $G$-module, then we shall consider the two induced cochain complexes

$$
\operatorname{Hom}_{G}(X, C) \text { and } \operatorname{Hom}(X, C)=\operatorname{Hom}_{z}(X, C)
$$

(for homological concepts see Chapter I of [7]). The first will be called the complex of equivariant cochains and its cohomology will be denoted by $H_{\mathrm{eq}}^{*}(X, C)$. The cohomology of $\operatorname{Hom}(X, C)$ will be denoted by $H^{*}(X, C)$.

For any symmetric $Q \subset G$ let us consider the scheme $\Gamma_{G}^{Q}$. Let

$$
\mathfrak{S}\left(\Gamma_{G}^{Q}\right)=\left\{\mathfrak{C}_{n}\left(\Gamma_{G}^{Q}\right), d\right\}_{n \geqq 0}
$$

be the complex of singular chains of $\Gamma_{G}^{Q}[7,3.2, \mathrm{p} .38]$. Then $\mho_{n}\left(\Gamma_{G}^{Q}\right)$ is the abelian group freely generated by the ordered $(n+1)$-tuples

$$
\left\langle g_{0}, g_{1}, \ldots, g_{n}\right\rangle \text {, where } g_{i} \in G \text { and } g_{i}^{-1} g_{j} \in Q \text { for all } i, j \text {. }
$$

We make each $\mathcal{夭}_{n}\left(\Gamma_{G}^{\odot}\right)$ into a $G$-module defining the action of $g \in G$ on a free generator by

$$
{ }^{g}\left\langle g_{0}, \ldots, g_{n}\right\rangle=\left\langle g g_{0}, g g_{1}, \ldots, g g_{n}\right\rangle \text {. }
$$

Then $\mathfrak{S}\left(\Gamma_{G}^{Q}\right)$ becomes a $G$-complex. The particular complex $\mathfrak{C}\left(\Gamma_{G}\right)=\mathfrak{C}\left(\Gamma_{G}^{G}\right)$ is the homogeneous $Z G$ resolution of $Z$ and can be used to obtain the cohomology of $G$; that is, if $C$ is any $G$-module, then [3, p. 52]

$$
H^{n}(G, C)=H_{\mathrm{eq}}^{n}\left(\mathscr{C}\left(\Gamma_{G}\right), C\right) .
$$

Let $V \subset G$ be symmetric. Then $\mathfrak{\subseteq}\left(\Gamma_{G}^{V}\right)$ is a subcomplex of $\mathfrak{\subseteq}\left(\Gamma_{G}\right)$ and we may consider the quotient complex $\mathfrak{C}^{(V)}\left(\Gamma_{G}\right)=\mathfrak{C}\left(\Gamma_{G}\right) / \mathfrak{C}\left(\Gamma_{G}^{V}\right)$. We define now

$$
H^{n}(V, C)=H_{\mathrm{eq}}^{n}\left(\mathfrak{S}\left(\Gamma_{G}^{V}\right), C\right), \quad H^{n}(G \bmod V, C)=H_{\mathrm{eq}}^{n}\left(\mathfrak{S}^{(V)}\left(\Gamma_{G}\right), C\right) .
$$

These will be referred to as the cohomology of $V$, or $G \bmod V$ with coefficients in $C$. The cohomology of $V$ can be defined for every local group $V$ (not necessarily enlargeable) such that there is a morphism of $V$ into the automorphism group of $C$. To see this, and for the purpose of applying $H^{2}(V, C), H^{2}(G \bmod V, C)$ and $H^{3}(G \bmod V, C)$ to local extensions, we need to redefine these groups in terms of another (the nonhomogeneous) complex.

Let $K_{G}=\left\{\left(K_{G}\right)_{n}, d\right\}_{n \geqq 0}$ be the chain complex associated with the standard inhomogeneous $Z G$-resolution of $Z$, i.e. $\left(K_{G}\right)_{n}$ is, as abelian group, freely generated by the $(n+1)$-tuples $\left\langle g_{0}, g_{1}, \ldots, g_{n}\right\rangle$ and $G$ acts on $\left(K_{G}\right)_{n}$ by permuting the generators according to the formula

$$
{ }^{g}\left\langle g_{0}, \ldots, g_{n}\right\rangle=\left\langle g g_{0}, g_{1}, \ldots, g_{n}\right\rangle
$$


The $G$-morphism $d$ is given by

$$
\begin{aligned}
d\left\langle g_{0}, g_{1}, \ldots, g_{n}\right\rangle= & \sum_{0 \leqq i<n}(-1)^{i}\left\langle g_{0}, \ldots, g_{i} g_{i+1}, \ldots, g_{n}\right\rangle \\
& +(-1)^{n}\left\langle g_{0}, g_{1}, \ldots, g_{n-1}\right\rangle .
\end{aligned}
$$

Then we have an isomorphism of complexes $\sigma: \mathfrak{S}\left(\Gamma_{G}\right) \rightarrow K_{G}$ defined on the generators of $\mathbb{E}_{n}\left(\Gamma_{G}\right)$ by

$$
\sigma_{n}\left\langle g_{0}, g_{1}, \ldots, g_{n}\right\rangle=\left\langle g_{0}, g_{0}^{-1} g_{1}, g_{1}^{-1} g_{2}, \ldots, g_{n-1}^{-1} g_{n}\right\rangle .
$$

Let $K_{G}^{V}$ be the image of $\Subset\left(\Gamma_{G}^{V}\right)$. Then it is easy to check that $\left(K_{G}^{V}\right)_{n}$ is the free abelian group with the free generators

$$
\left\langle g_{0}, g_{1}, \ldots, g_{n}\right\rangle \text {, where } g_{i} g_{i+1} \cdots g_{l} \in V \text { for every } 0<i \leqq l \leqq n .
$$

We denote by $K_{G}^{(V)}$ the quotient complex $K_{G} / K_{G}^{V}$. Then the cohomology of $V$ and $G \bmod V$ can be written as

$$
H^{n}(V, C)=H_{\mathrm{eq}}^{n}\left(K_{G}^{V}, C\right), H^{n}(G \bmod V, C)=H_{\mathrm{eq}}^{n}\left(K_{G}^{(V)}, C\right) .
$$

We adopt the following convention: for any $C$-valued $n$-dimensional equivariant cochain $f$ on $K_{G}$ we denote by $f\left[g_{1}, \ldots, g_{n}\right]$ the value which $f$ takes on $\left\langle 1, g_{1}, \ldots, g_{n}\right\rangle$. Since $\left(K_{G}\right)_{n}$ is free, as $G$-module over the generators $\left\langle 1, g_{1}, \ldots, g_{n}\right\rangle, f$ is uniquely described by all the elements $f\left[g_{1}, \ldots, g_{n}\right] \in C\left(g_{i} \in G\right)$ and these can be chosen arbitrarily. From this convention and (4.1) follows the coboundary formula

$$
\begin{aligned}
(\delta f)\left[g_{1}, \ldots, g_{n+1}\right]={ }^{g_{1}} f\left[g_{2}, \ldots, g_{n+1}\right] \\
\quad+\sum_{1 \leqq i \leqq n}(-1)^{i} f\left[g_{1}, \ldots, g_{i} g_{i+1}, \ldots, g_{n+1}\right]+(-1)^{n+1} f\left[g_{1}, \ldots, g_{n}\right]
\end{aligned}
$$

(we denote the action of $g$ on $C$ by $c \rightarrow{ }^{g} c$ ).

If $V$ is a local group, let us say that an abelian group $C$ is a $V$-module if we have a morphism of $V$ into the automorphism group of $C$. Let us denote by $\left(A_{V}\right)_{n}$ the abelian group freely generated by the $n$-tuples

$$
\left\langle g_{1}, g_{2}, \ldots, g_{n}\right\rangle \text {, where } g_{i} g_{i+1} \cdots g_{l} \text { exists in } V \text { for all } 1 \leqq i \leqq l \leqq n,
$$

and let us call every morphism (of abelian groups) $f:\left(A_{V}\right)_{n} \rightarrow C$ an $n$-dimensional equivariant $V$-cochain. We define the coboundary $\delta f$ of $f$ to be the $(n+1)$-dimensional equivariant $V$-cochain given by (4.3). It is easy to see from (4.2) that if $V \subset G$, then the equivariant $V$-cochains are precisely the equivariant cochains on $K_{G}^{V}$, and the coboundary operators coincide. Hence, if we redefine $H^{*}(V, C)$ to be the group of $V$-cocycles modulo the subgroup of $V$-coboundaries, we shall get the same object as defined previously for the case when $V \subset G$. (It is also possible to obtain $H^{*}(V, C)$ as a derived functor in a suitable category, see [16].)

5. The cohomology of a $G$-complex. For a $G$-module $A$ let us denote by $A^{G}$ the submodule of $G$-stable (invariant under the action of $G$ ) elements of $A$. Let $Y=\left\{Y^{q}, d\right\}_{q \geqq 0}$ denote a cochain $G$-complex; then it is easy to see that

$$
Y^{G}=\left\{\left(Y^{q}\right)^{G}, d\right\}_{q \geqq 0}
$$


is a subcomplex of $Y$ so that we have a morphism $\beta^{*}: H^{*}\left(Y^{G}\right) \rightarrow H^{*}(Y)$ induced by the inclusion $Y^{G} \subset Y$. The cohomology group $H^{*}(Y)$ inherits a $G$-structure from $Y$ and it is clear that the image under the above morphism consists of $G$ stable elements of $H^{*}(Y)$. So finally we have a morphism

$$
\beta^{*}: H^{*}\left(Y^{G}\right) \rightarrow\left(H^{*}(Y)\right)^{G}
$$

induced by $Y^{G} \subset Y$. The results of $\$ 10,11$ and 12 are based on the following

Lemma. If $Y$ is acyclic in dimensions $0,1, \ldots, n-1$ and

$$
H^{p}\left(G, Y^{q}\right)=0 \text { for all } p>0, q \geqq 0
$$

then $\beta^{n}: H^{n}\left(Y^{G}\right) \rightarrow\left(H^{n}(Y)\right)^{G}$ is an isomorphism.

Proof. Let $X=\left\{X^{p, q}, d^{\prime}, d^{\prime \prime}\right\}_{p, q \geqq 0}$ be the bicomplex, where

$$
X^{p, q}=\operatorname{Hom}_{G}\left(\left(K_{G}\right)_{p}, Y^{q}\right)
$$

and $d^{\prime}: X^{p, q} \rightarrow X^{p+1, q}, d^{\prime \prime}: X^{p, q} \rightarrow X^{p, q+1}$ are induced by the boundary operator in $K_{G}$ (see previous section) and the coboundary operator in $Y$ (see [7, Chapter I, 2.8, p. 33]). We put $X^{n}=\bigoplus_{p+q=n} X^{p, q}$ and define $d: X^{n} \rightarrow X^{n+1}$ by setting $d=d^{\prime}+d^{\prime \prime}$ on each $X^{p, q}$. Then $\left\{X^{n}, d\right\}_{n \geqq 0}$ is a cochain complex; we shall denote it by Tot $X$.

We now identify $X^{0, q}$ in the natural way with $Y^{q}$ and observe that $d^{\prime}=0$ on $\left(Y^{q}\right)^{G} \subset X^{0, q}$, so that $Y^{G}$ is a subcomplex of Tot $X$. The inclusion induces morphisms

$$
H^{q}\left(Y^{q}\right) \rightarrow H^{q}(\operatorname{Tot} X) ; \quad q=0,1,2, \ldots
$$

It is shown in [9, Proposition 4, p. 114] that if (5.1) holds, then (5.2) are isomorphisms. Alternatively this can be proved by taking the spectral sequence associated to the second filtration of Tot $X$ and observing that it collapses, by (5.1). (See "Lyndon spectral sequence" in [11, p. 351].)

Let $\left\{E_{r}^{p, q}\right\}$ be the spectral sequence of Tot $X$ associated to the first filtration ([7, 4.8, p. 86]). Then

$$
{ }^{\prime} E_{2}^{p, q}=H^{p}\left(G, H^{q}(Y)\right)
$$

[9, Lemma 2, p. 116]. Since $Y$ is acyclic in dimensions $0,1, \ldots, n-1$, it follows that ' $E_{2}^{p, q}=0$ for $q=0,1, \ldots, n-1$ and all $p \geqq 0$. This implies

$$
{ }^{\prime} E_{\infty}^{0, n}={ }^{\prime} E_{2}^{0, n} \text { and }{ }^{\prime} E_{\infty}^{p, q}=0 \text { for } q=0,1, \ldots, n-1 \text { and all } p .
$$

Consequently the natural epimorphism

$$
H^{n}(\operatorname{Tot} X) \rightarrow^{\prime} E_{\infty}^{0, n}
$$

is in this case an isomorphism.

Combining (5.2), (5.4) and (5.3),

$$
H^{n}\left(Y^{G}\right) \rightarrow H^{n}(\operatorname{Tot} X) \rightarrow^{\prime} E_{\infty}^{0, n}={ }^{\prime} E_{2}^{0, n}=H^{0}\left(G, H^{n}(Y)\right)=\left(H^{n}(Y)\right)^{G},
$$


we get an isomorphism $H^{n}\left(Y^{G}\right) \rightarrow\left(H^{n}(Y)\right)^{G}$. We have to check that it induced by $Y^{G} \subset Y$. Take $\xi \in\left(Y^{n}\right)^{G} \subset X^{0, n}$ such that $d \xi=0$. It will suffice to show that the image of $\xi$ under (5.5) is the cohomology class $\{\xi\} \in H^{n}(Y)$. By the inclusion $Y^{G} \subset$ Tot $X, \xi$ is identified with the cocycle

$$
\xi \in X^{0, n}=\operatorname{Hom}_{G}\left(\left(K_{G}\right)_{0}, Y^{n}\right)
$$

such that $\xi\left\langle g_{0}\right\rangle=\xi$ for all $\left\langle g_{0}\right\rangle$ in $\left(K_{G}\right)_{0}$. Let $\xi^{0}$ be the equivalence class of $\xi$ in $' E_{1}^{0, n}$. We have $\xi^{0} \in E_{1}^{0, n}={ }^{\prime \prime} H^{n}\left(\operatorname{Hom}_{G}\left(\left(K_{G}\right)_{0}, Y\right)\right)=\operatorname{Hom}_{G}\left(\left(K_{G}\right)_{0}, H^{n}(Y)\right.$ ) (see $\left[9\right.$, Lemma 1, p. 115]) and $\xi^{0}\left\langle g_{0}\right\rangle=\{\xi\} \in H^{n}(Y)$ for all $\left\langle g_{0}\right\rangle$ in $\left(K_{G}\right)_{0}$. Clearly $\{\xi\} \in\left(H^{n}(Y)\right)^{G}$, whence it follows that

$$
\xi^{0} \in E^{\prime} E_{2}^{0, n}={ }^{\prime} H^{0}\left(\operatorname{Hom}_{G}\left(K_{G}, H^{n}(Y)\right)\right)=H^{0}\left(G, H^{n}(Y)\right) .
$$

Finally, in the identification $H^{0}\left(G, H^{n}(Y)\right)=\left(H^{n}(Y)\right)^{G}$, the element identified with $\xi^{0}$ is the cohomology class $\{\xi\} \in H^{n}(Y)$. This completes the proof.

6. Basic isomorphisms. Let us recall that if $A, B$ are two $G$-modules then $\operatorname{Hom}(A, B)$ can be made into a $G$-module by defining the action of $g$ on $f \in \operatorname{Hom}(A, B)$ by

$$
\left({ }^{g} f\right)(a)={ }^{g^{-1}}\left(f\left({ }^{g} a\right)\right) \text { for each } a \text { in } A .
$$

Then, if $X$ is a chain $G$-complex, $C$ is a $G$-module, and $Y$ is the cochain complex $\operatorname{Hom}(X, C)$, we have that $Y$ is a $G$-complex, moreover $Y^{G}=\operatorname{Hom}_{G}(X, C)$. Applying this to $X=K_{G}^{(V)}$, we shall deduce from the previous section the

LEMMA 1. Let $n \geqq 2$. If $H_{0}\left(K_{G}^{V}\right)=Z$, and if $n>2, H_{i}\left(K_{G}^{V}\right)=0$ for $i=1,2, \ldots, n-2$, then the inclusion of cochain complexes $\operatorname{Hom}_{G}\left(K_{G}^{(V)}, C\right) \subset \operatorname{Hom}\left(K_{G}^{(V)}, C\right)$ induces an isomorphism

$$
\beta^{n}: H^{n}(G \bmod V, C) \rightarrow\left(H^{n}\left(K_{G}^{(V)}, C\right)\right)^{G} .
$$

REMARK. Since $K_{G}^{V}$ is isomorphic to $\subseteq\left(\Gamma_{G}^{V}\right)$ the assumption of the lemma is equivalent to: $V$ generates $G$ (i.e. $H_{0}\left(\Gamma_{G}^{V}\right)=Z$ ) and $\subseteq\left(\Gamma_{G}^{V}\right)$ is acyclic in dimensions $1,2, \ldots$, $n-2$. In case $n=3$ this will certainly be satisfied when $G$ is $V$-monodrome (see Lemma 2 of $\S 3$, p. 9).

For the proof denote by $Y$ the cochain $G$-complex $\operatorname{Hom}\left(K_{G}^{(V)}, C\right)$. The result follows from the previous section provided we show that

$$
\begin{gathered}
H^{p}\left(G, \operatorname{Hom}\left(\left(K_{G}^{(V)}\right)_{q}, C\right)\right)=0 \text { for all } p>0 \text { and } q \geqq 0, \\
H^{i}\left(K_{G}^{(V)}, C\right)=0 \text { for } i=0,1, \ldots, n-1 .
\end{gathered}
$$

We observe that in the exact sequence

$$
E_{G}^{V}: 0 \rightarrow K_{G}^{V} \subset K_{G} \rightarrow K_{G}^{(V)} \rightarrow 0,
$$

$K_{G}$ splits as the direct sum of $K_{G}^{V}$ and another free $G$-module, thus $K_{G}^{(V)}$ is free and (6.1) follows from known results (see [9, Corollary on p. 116] also [11, Lemma 9.3 , p. 350]). 
To prove (6.2) let us first note that $H^{0}\left(K_{G}^{(V)}, C\right)=0$ by $\left(K_{G}^{(V)}\right)_{0}=\left(K_{G}\right)_{0} /\left(K_{G}^{V}\right)_{0}=0$. Now let

$$
\begin{aligned}
\cdots \longrightarrow H^{i-1}\left(K_{G}, C\right) & \longrightarrow H^{i-1}\left(K_{G}^{V}, C\right) \\
& \stackrel{\partial_{E}}{\longrightarrow} H^{i}\left(K_{G}^{(V)}, C\right) \longrightarrow H^{i}\left(K_{G}, C\right) \longrightarrow \cdots
\end{aligned}
$$

be the exact cohomology sequence of $E_{G}^{V}$. Since $H^{1}\left(K_{G}, C\right)=0, H^{1}\left(K_{G}^{(V)}, C\right)=0$ will follow provided we show that $H^{0}\left(K_{G}, C\right) \rightarrow H^{0}\left(K_{G}^{V}, C\right)$, induced by $K_{G}^{V} \subset K_{G}$ is an isomorphism. Since $\left(K_{G}^{V}\right)_{0}=\left(K_{G}\right)_{0}$, it is enough to verify that every boundary in $\left(K_{G}\right)_{0}$ is a boundary in $\left(K_{G}^{V}\right)_{0}$. But if this were not so, $K_{G}^{V} \subset K_{G}$ would induce an epimorphism $Z=H_{0}\left(K_{G}^{V}\right) \rightarrow H_{0}\left(K_{G}\right)=Z$ with nontrivial kernel. a contradiction. Thus $H^{1}\left(K_{G}^{(V)}, C\right)=0$.

For $2 \leqq i \leqq n-1$, the exact cohomology sequence gives $H^{i}\left(K_{G}^{(V)}, C\right) \simeq H^{i-1}\left(K_{G}^{V}, C\right)$, so we should show that for those $i, H^{i-1}\left(K_{G}^{V}, C\right)=0$. This follows from the universal coefficient theorem

$$
0 \longrightarrow \operatorname{Ext}^{1}\left(H_{i-2}\left(K_{G}^{V}\right), C\right) \longrightarrow H^{i-1}\left(K_{G}^{V}, C\right) \underset{\gamma}{\longrightarrow} \operatorname{Hom}\left(H_{i-1}\left(K_{G}^{V}\right), C\right) \longrightarrow 0
$$

since the second and fourth terms vanish, for we have by assumption $H_{i-2}\left(K_{G}^{V}\right)=Z$ or 0 and $H_{i-1}\left(K_{G}^{v}\right)=0$. This completes the proof of Lemma 1 .

Since $K_{G}$ is acyclic, it follows that $\partial_{E}$ in (6.4) is for $i>1$ an isomorphism. It is easy to check that $\partial_{E}$ is a $G$-morphism, hence we have for $i>1$ an isomorphism

$$
\partial_{\bar{E}}^{-1}:\left(H^{i}\left(K_{G}^{(V)}, C\right)\right)^{G} \rightarrow\left(H^{i-1}\left(K_{G}^{V}, C\right)\right)^{G} .
$$

Also $\gamma$ in (6.5) is readily seen to be a $G$-morphism, so we have a morphism

$$
\gamma:\left(H^{i-1}\left(K_{G}^{V}, C\right)\right)^{G} \rightarrow \operatorname{Hom}_{G}\left(H_{i-1}\left(K_{G}^{V}\right), C\right) .
$$

LEMMA 2. Let $n \geqq 2$. If $H_{0}\left(K_{G}^{V}\right)=Z$, and if $n>2, H_{i}\left(K_{G}^{V}\right)=0$ for $i=1,2, \ldots, n-2$, then the composite

$$
\kappa: H^{n}(G \bmod V, C) \stackrel{{ }^{\gamma \circ \partial_{E}^{-1}} \stackrel{\beta^{n}}{\longrightarrow}}{\longrightarrow} \operatorname{Hom}_{G}\left(H_{n-1}\left(K_{G}^{v}\right), C\right)
$$

is an isomorphism.

The only thing to check is that $\gamma$ is an isomorphism. But this follows from (6.5) taken with $i=n$.

We shall need an invariance property of $\kappa$ under "change of groups". Suppose $\omega: \hat{G} \rightarrow G$ is a morphism of groups, then every $G$-module $B$ becomes a $\hat{G}$-module by

$$
{ }^{\hat{g}} b={ }^{\omega(\hat{g})} b \text { for every } b \in B, \hat{g} \in \hat{G} .
$$

Now let $A$ be a $\hat{G}$-module, let $B$ be a $G$-module and let $A \rightarrow B$ be a $\hat{G}$-morphism. Then, for every abelian group $C$ we have the induced morphism $\operatorname{Hom}(B, C)$ $\rightarrow \operatorname{Hom}(A, C)$. It is not difficult to check that if $C$ is a $G$-module, then the image of $\operatorname{Hom}_{G}(B, C)$ is contained in $\operatorname{Hom}_{\hat{G}}(A, C)$. 
Let a morphism $\omega:(\hat{V}, \hat{G}) \rightarrow(V, G)$ be given. Then we have a $\hat{G}$-morphism of exact sequences $E_{G \wedge}^{V \wedge} \rightarrow E_{G}^{V}$ (see (6.3)) given on $K_{\hat{G}}$ by

$$
\left\langle\hat{g}_{0}, \hat{g}_{1}, \ldots, \hat{g}_{n}\right\rangle \rightarrow\left\langle\omega \hat{g}_{0}, \omega \hat{g}_{1}, \ldots, \omega \hat{g}_{n}\right\rangle .
$$

In particular $K_{G \wedge}^{\left(V^{\wedge}\right)} \rightarrow K_{G}^{(V)}$ induces for any abelian group $C$ a morphism of cochain complexes $\operatorname{Hom}\left(K_{G}^{(V)}, C\right) \rightarrow \operatorname{Hom}\left(K_{G \wedge}^{(V \wedge)}, C\right)$. If $C$ is a $G$-module then, by our previous remark, the subcomplex $\operatorname{Hom}_{G}\left(K_{G}^{(V)}, C\right)$ is mapped into $\operatorname{Hom}_{\hat{G}}\left(K_{G \wedge}^{\left(V^{\wedge}\right)}, C\right)$. We have therefore the induced morphisms

$\omega^{*}: H^{*}\left(K_{G}^{(V)}, C\right) \rightarrow H^{*}\left(K_{G \wedge}^{(V \wedge)}, C\right), \quad \omega_{\mathrm{eq}}^{*}: H^{*}(G \bmod V, C) \rightarrow H^{*}(\hat{G} \bmod \hat{V}, C)$. $\omega:(\hat{V}, \hat{G}) \rightarrow(V, G)$ also gives a $\hat{G}$-morphism $\omega_{*}: H_{*}\left(K_{G \wedge}^{V \wedge}\right) \rightarrow H_{*}\left(K_{G}^{V}\right)$ which induces

$$
\bar{\omega}_{*}: \operatorname{Hom}\left(H_{*}\left(K_{G}^{V}\right), C\right) \rightarrow \operatorname{Hom}\left(H_{*}\left(K_{G \wedge}^{V \wedge}\right), C\right) .
$$

LEMMA 3. If $n \geqq 2$, then $\bar{\omega}_{n-1} \circ \kappa=\hat{\kappa} \circ \omega_{\mathrm{eq}}^{n}$.

The proof follows from the commutativity of the diagram

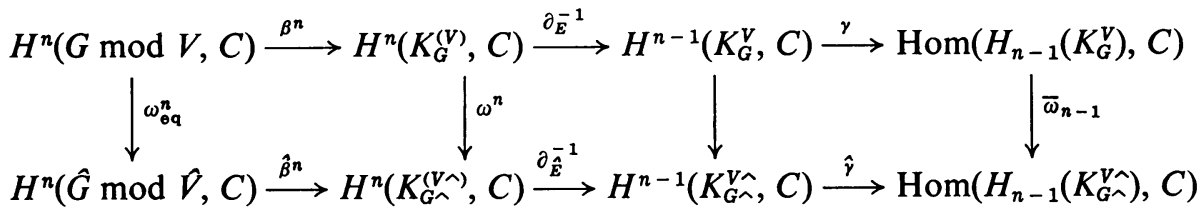

which is checked on each square separately. It suffices to recall that the image of $\operatorname{Hom}_{G}\left(H_{n-1}\left(K_{G}^{V}\right), C\right)$ under $\bar{\omega}_{n-1}$ is in $\operatorname{Hom}_{\hat{G}}\left(H_{n-1}\left(K_{G \wedge}^{V \wedge}\right), C\right)$.

7. Multiplicators and abstract kernels. Let $A(N), I(N)$ denote the group of automorphisms and the subgroup of inner automorphisms of $N$, let further $\iota(n): N \rightarrow N$ be the inner automorphism $x \rightarrow n x n^{-1}$.

Let $V$ be a local group and let $N$ be a group. If we have a map $\alpha: V \rightarrow A(N)$ (not necessarily a morphism) and a function $h$ assigning to every $g_{1}, g_{2} \in V$, such that $g_{1} g_{2}$ exists, an element $h\left(g_{1}, g_{2}\right)$ of $N$, then we shall call the pair $\langle\alpha, h\rangle$ a premultiplicator from $V$ to $N$ if $h(1,1)=1$ and

$$
\alpha\left(g_{1}\right) \alpha\left(g_{2}\right)=\iota\left(h\left(g_{1}, g_{2}\right)\right) \alpha\left(g_{1} g_{2}\right)
$$

holds whenever $g_{1}, g_{2}, g_{1} g_{2} \in V$.

Let us call a morphism $\Theta: V \rightarrow A(N) / I(N)$ an abstract kernel and denote it shortly by $\langle V, N, \Theta\rangle$. Given a premultiplicator $\langle\alpha, h\rangle$ from $V$ to $N$ and an abstract kernel $\langle V, N, \Theta\rangle$ we shall call $\langle\alpha, h\rangle$ a premultiplicator of $\langle V, N, \Theta\rangle$, and $\langle V, N, \Theta\rangle$ the kernel of $\langle\alpha, h\rangle$ if $\alpha$ factorizes $\langle V, N, \Theta\rangle$, that is when

$$
\Theta: V \stackrel{\alpha}{\longrightarrow} A(N) \longrightarrow A(N) / I(N),
$$

where the last map is the quotient-morphism. Clearly the abstract kernel of a premultiplicator $\langle\alpha, h\rangle$ is defined by (7.2). A premultiplicator of an abstract kernel is obtained by choosing $\alpha$ such that (7.2) holds and then $h$ satisfying (7.1). 
Let $\langle\alpha, h\rangle$ be a premultiplicator from $V$ to $N$, let $\langle V, N, \Theta\rangle$ be the abstract kernel of $\langle\alpha, h\rangle$, and let $C$ be the centre of $N$. Then $C$ is a $V$-module by

$$
\Theta: V \rightarrow A(N) / I(N) \rightarrow A(C) .
$$

(Though $C$ is abelian, we shall write it multiplicatively, denoting the action of $V$ by ${ }^{v} c$.) For any $g_{1}, g_{2}, g_{3}$ in $V$ such that $g_{1} g_{2}, g_{2} g_{3}, g_{1} g_{2} g_{3}$ exist, let us denote

$$
f_{\alpha, h}\left[g_{1}, g_{2}, g_{3}\right]={ }^{\alpha\left(g_{1}\right)} h\left(g_{2}, g_{3}\right) h\left(g_{1}, g_{2} g_{3}\right)\left(h\left(g_{1} g_{2}, g_{3}\right)\right)^{-1}\left(h\left(g_{1}, g_{2}\right)\right)^{-1} \text {. }
$$

Lemma 1. $f_{\alpha, h}$ is a $C$-valued equivariant 3-dimensional V-cocycle. Moreover, if $\langle\alpha, h\rangle,\left\langle\alpha^{\prime}, h^{\prime}\right\rangle$ are premultiplicators of the same abstract kernel, then $f_{\alpha, h}\left(f_{\alpha^{\prime}, h^{\prime}}\right)^{-1}$ is a $V$-coboundary.

This is well known if $V$ is a group and the proof in [3, Lemma 7.2, p. 332] goes through for a local group. Given an abstract kernel $\langle V, N, \Theta\rangle$, we have from the second part of the lemma that the cohomology class $\left\{f_{\alpha, h}\right\}$ does not depend on the premultiplicator associated to it. We define

$$
\operatorname{Obs}\langle V, N, \Theta\rangle=\left\{f_{\alpha, h}\right\} \in H^{3}(V, C)
$$

and we call $\left\{f_{\alpha, h}\right\}$ the obstruction of the abstract kernel $\langle V, N, \Theta\rangle$ (cf. Lemma 2 below).

A premultiplicator $\langle\alpha, h\rangle$ will be called a multiplicator if $f_{\alpha, h}=1$ identically. Given a multiplicator $\langle\alpha, h\rangle$ from $V$ to $N$, we define a local.extension

$$
\mathfrak{L}: 1 \longrightarrow N \subset U \underset{\phi}{\longrightarrow} V \longrightarrow 1
$$

as follows. We set $U=N \times V$ and define the product in $U$ by

$$
\left\langle n_{1}, g_{1}\right\rangle\left\langle n_{2}, g_{2}\right\rangle=\left\langle n_{1}^{\alpha\left(g_{1}\right)} n_{2} h\left(g_{1}, g_{2}\right), g_{1} g_{2}\right\rangle,
$$

so that the product exists iff $g_{1} g_{2}$ exists. Then the map $U \rightarrow_{\phi} V$ given by $\phi\langle n, g\rangle=g$ is a strong epimorphism with kernel $N \times 1$. Moreover, by $h(1,1)=1, N \times 1$ is isomorphic to $N$; identifying it with $N$ we get $\mathfrak{R}$.

If $\mathfrak{L}$ is a local extension, let us call a map $\psi: V \rightarrow U$ a $\phi$-selector if $\psi(1)=1$ and $\phi(\psi(g))=g$ for all $g$ in $V$. Let us denote by $\iota_{N}: U \rightarrow A(N)$ the morphism which assigns to $u \in U$ the automorphism $n \rightarrow u n u^{-1}$ of $N$. Having chosen a $\phi$-selector $\psi$ we put

$$
\alpha: V \underset{\psi}{\longrightarrow} \underset{\iota_{N}}{\longrightarrow} A(N)
$$

and observe that if $g_{1}, g_{2} \in V$ are such that $g_{1} g_{2}$ exists, then there is an $h\left(g_{1}, g_{2}\right)$ in $N$ satisfying

$$
\psi\left(g_{1}\right) \psi\left(g_{2}\right)=h\left(g_{1}, g_{2}\right) \psi\left(g_{1} g_{2}\right)
$$

The pair $\langle\alpha, h\rangle$ obtained in this way is a multiplicator ((7.1) follows from (7.4) by applying $\iota_{N}$ and $f_{\alpha, h}=1$ is obtained by evaluating $\psi\left(g_{1}\right) \psi\left(g_{2}\right) \psi\left(g_{3}\right)$ with the aid of 
(7.4)). We call $\langle\alpha, h\rangle$ the multiplicator defined by $\mathfrak{Q}$ and $\psi$, or simply a multiplicator of $\mathfrak{R}$. We observe that

(a) if $\langle\alpha, h\rangle$ is defined by $\mathfrak{Q}$ and $\psi$, then $\langle\alpha, h\rangle$ defines an extension isomorphic to $\mathfrak{R}$, the isomorphism being $\langle n, g\rangle \rightarrow n \psi(g)$ on $N \times V$ and identity on $V$,

(b) if $\langle\alpha, h\rangle$ defines $\mathfrak{I}$, then $\langle\alpha, h\rangle$ is defined by $\mathfrak{L}$ and the $\phi$-selector $\psi(g)=\langle 1, g\rangle$. Given an extension $\mathfrak{L}$ as above, we choose a $\phi$-selector $\psi: V \rightarrow U$ and set

$$
\Theta: V \underset{\psi}{\longrightarrow} \underset{\iota_{N}}{\longrightarrow} A(N) \longrightarrow A(N) / I(N) .
$$

It is easily checked that $\Theta$ does not depend on the choice of $\psi$ and that $\Theta$ is a morphism, so we have an abstract kernel $\langle V, N, \Theta\rangle$. We shall call it the abstract kernel of $\mathfrak{L}$. If $\langle\alpha, h\rangle$ is a multiplicator of $\mathfrak{R}$, then it follows by (7.3) and (7.2) that $\langle\alpha, h\rangle$ and $\mathfrak{Q}$ have the same abstract kernel.

LEMMA 2. There exists an extension $\mathfrak{Q}$ whose kernel is $\langle V, N, \Theta\rangle$ if and only if $\operatorname{Obs}\langle V, N, \Theta\rangle=0$.

Although the proof is nearly the same as the one for groups [3, Theorem 8.1, p. 333] we carry it through because we use nonnormalized cochains.

The condition is clearly necessary; the abstract kernel of an extension is the kernel of a multiplicator, so its obstruction must vanish.

Now suppose $\operatorname{Obs}\langle V, N, \Theta\rangle=0$, and let $\langle\alpha, h\rangle$ be a premultiplicator of $\langle V, N, \Theta\rangle$. Then the cohomology class $\left\{f_{\alpha, h}\right\} \in H^{3}(V, C)$ vanishes, i.e. we have a $V$-cochain $c$ of dimension 2 such that $f_{\alpha, h}=\delta c$. Let $s$ be a 1-dimensional $V$-cochain such that $(c[1,1])^{-1}=(\delta s)[1,1]$, such a cochain $s$ does always exist by $\delta(s)[1,1]=s[1]$ (see (4.3)). Consider the cochain $c^{\prime}=(\delta s) \cdot c$. Then $f_{\alpha, h}=\delta c^{\prime}$ and $c^{\prime}[1,1]=1$. It follows that if we put $h^{\prime}=\left(c^{\prime}\right)^{-1} \cdot h$, then $f_{\alpha, h^{\prime}}=\left(\delta c^{\prime}\right)^{-1} f_{\alpha, h}=1$, so that $\left\langle\alpha, h^{\prime}\right\rangle$ is a multiplicator. Let $\mathfrak{Q}$ be the extension defined by $\left\langle\alpha, h^{\prime}\right\rangle$. Then $\left\langle\alpha, h^{\prime}\right\rangle$ is a multiplicator of $\mathfrak{L}$ (by $(b)$ above), and hence the kernel of $\mathfrak{L}$ coincides with the kernel of $\left\langle\alpha, h^{\prime}\right\rangle$, i.e. with $\langle V, N, \Theta\rangle$. This completes the proof.

Let $G$ be a group containing $V$. If $\langle\alpha, h\rangle$ is a premultiplicator from $G$ to $N$, we shall denote by $\langle a, h\rangle \mid V$ its restriction to $V$, i.e. $\langle\alpha, h\rangle \mid V=\left\langle\alpha \mid V, h_{V}\right\rangle$ where

$$
h_{V}\left(g_{1}, g_{2}\right)=h\left(g_{1}, g_{2}\right) \text { if } g_{1}, g_{2}, g_{1} g_{2} \in V
$$

and is not defined otherwise. A premultiplicator from $V$ to $N$ which is the restriction of a premultiplicator from $G$ to $N$ will be called enlargeable over $G$.

The following lemma collects various auxiliary results which will be needed later.

LEMma 3. Suppose that

(i) $\mathfrak{L}: 1 \rightarrow N \subset U \rightarrow_{\phi} V \rightarrow 1$ is a local extension,

(ii) $G$ is a group containing $V$,

(iii) $\langle\alpha, h\rangle$ is a premultiplicator from $G$ to $N$ such that $\langle\alpha, h\rangle \mid V$ is a multiplicator of $\mathfrak{L}$,

(iv) $\langle G, N, \Theta\rangle$ is the abstract kernel of $\langle\alpha, h\rangle$, 
(v) $\alpha^{\prime}: G \rightarrow A(N)$ satisfies $\alpha^{\prime}(1)=1$ and factorizes $\langle G, N, \Theta\rangle$ i.e.

$$
\Theta: G \stackrel{\alpha^{\prime}}{\longrightarrow} A(N) \longrightarrow A(N) / I(N) .
$$

Then there exists a premultiplicator $\left\langle\alpha^{\prime}, h^{\prime}\right\rangle$ from $G$ to $N$ such that

(j) $f_{\alpha^{\prime}, h^{\prime}}=f_{\alpha, h}$,

(jj) $\left\langle\alpha^{\prime}, h^{\prime}\right\rangle \mid V$ is a multiplicator of $\mathrm{R}$.

If it is further to (i), .., (v) assumed that $\langle\alpha, h\rangle$ is a multiplicator of some global extension (5), then the above $\left\langle\alpha^{\prime}, h^{\prime}\right\rangle$ is also a multiplicator of \&5. And if moreover $\alpha=\alpha^{\prime}$ on $V$, then $\langle\alpha, h\rangle \mid V$ and $\left\langle\alpha^{\prime}, h^{\prime}\right\rangle \mid V$ both are defined by $\mathfrak{L}$ and the same $\phi$ selector.

Proof. It follows from (7.5) that for each $g \in G$ we can find some $k(g) \in N$ such that $\alpha^{\prime}(g)=\imath(k(g)) \alpha(g)$. If we have $\alpha^{\prime}=\alpha$ on $V$, then let us choose $k=1$ on $V$. We choose $k(1)=1$. We put

$$
h^{\prime}\left(g_{1}, g_{2}\right)=k\left(g_{1}\right)^{\alpha\left(g_{1}\right)} k\left(g_{2}\right) h\left(g_{1}, g_{2}\right) k^{-1}\left(g_{1} g_{2}\right) \text {. }
$$

We assert that $\left\langle\alpha^{\prime}, h^{\prime}\right\rangle$ is the required premultiplicator from $G$ to $N$. The premultiplicator property is easily checked and the equality $f_{\alpha, h}=f_{\alpha^{\prime}, h^{\prime}}$ follows by a somewhat lengthy computation (see [3, proof of Lemma 7.3; p. 332]). Moreover, if $\langle\alpha, h\rangle \mid V$ (or $\langle\alpha, h\rangle$ ) is a multiplicator defined by $\mathfrak{L}$ (or by a global extension (S) and $\psi$, then $\left\langle\alpha^{\prime}, h^{\prime}\right\rangle \mid V$ (or $\left\langle\alpha^{\prime}, h^{\prime}\right\rangle$ ) is the multiplicator defined by $\mathfrak{L}$ (or (S)) and $\psi^{\prime}$, where $\psi^{\prime}(g)=k(g) \psi(g)$ for all $g$ in $V$ (or in $G$ ). If $\alpha=\alpha^{\prime}$ on $V$, then clearly $\psi=\psi^{\prime}$ on $V$.

8. The obstruction of $\langle G, N, \Theta\rangle$ over $\mathfrak{L}$. Let $\mathfrak{L}: 1 \rightarrow N \subset U \rightarrow_{\phi} V \rightarrow 1$ be a local extension such that $V$ is contained in a group $G$. We denote by $\left\langle V, N, \Theta_{V}\right\rangle$ the kernel of $\mathfrak{L}$ and by $C$ the centre of $N$. Then every abstract kernel $\langle G, N, \Theta\rangle$ which is an enlargement of $\left\langle V, N, \Theta_{V}\right\rangle$ (i.e. such that $\Theta \mid V=\Theta_{V}$ ) induces a $G$ module structure on $C$ and we can consider the corresponding group

$$
H^{3}(G \bmod V, C) \text {. }
$$

THEOREM. For every extension $\mathfrak{L}$ as above and every enlargement $\langle G, N, \Theta\rangle$ of the kernel of $\mathfrak{Q}$, there exists an element

$$
\operatorname{Obs} \Omega\langle G, N, \Theta\rangle \in H^{3}(G \bmod V, C)
$$

which vanishes if and only if $\langle G, N, \Theta\rangle$ is the kernel of some global enlargement $\mathrm{SS}$ of $\mathfrak{L}$. If $C$ is an arbitrary $G$-module and $V$ is a symmetric subset of $G$ then for each $a \in H^{3}(G \bmod V, C)$ there exists an extension $\mathfrak{L}$ over $V$ such that $C=$ centre $N$, and whose abstract kernel is enlargeable to a kernel $\langle G, N, \Theta\rangle$ for which

$$
a=\operatorname{Obs} \mathfrak{R}\langle G, N, \Theta\rangle \text {. }
$$

Proof. Since the abstract kernel of $\mathfrak{Q}$ is enlargeable to $\langle G, N, \Theta\rangle$, every multiplicator of $\mathfrak{L}$ is enlargeable to a premultiplicator of $\langle G, N, \Theta\rangle$. Let us call a premultiplicator $\langle\alpha, h\rangle$ of $\langle G, N, \Theta\rangle$ admissible if $\langle\alpha, h\rangle \mid V$ is a multiplicator of $\mathfrak{R}$. 
If $\langle\alpha, h\rangle$ is admissible, then $f_{\alpha, h} \mid\left(K_{G}^{V}\right)_{2}=1$, so $f_{\alpha, h}$ can be regarded as equivariant cocycle on $K_{G}^{(V)}$. Let us show that its cohomology class $\left\{f_{\alpha, h}\right\}$ in $H^{3}(G \bmod V, C)$ does not depend on the choice of $\langle\alpha, h\rangle$. For this purpose it will be enough to prove the assertions

I. If $\langle\alpha, h\rangle,\left\langle\alpha, h^{\prime}\right\rangle$ are admissible, then there exists an equivariant cochain $c$ on $K_{G}^{(V)}$ such that

$$
f_{\alpha, h^{\prime}}\left(f_{\alpha, h}\right)^{-1}=\delta c .
$$

II. If $\langle\alpha, h\rangle,\left\langle\alpha^{\prime}, h^{\prime \prime}\right\rangle$ are admissible, then there exists an admissible $\left\langle\alpha^{\prime}, h^{\prime}\right\rangle$ satisfying $f_{\alpha, h}=f_{\alpha^{\prime}, h^{\prime}}$

Proof of I. Let $\psi, \psi^{\prime}$ denote the $\phi$-selectors which define the multiplicators $\langle\alpha, h\rangle\left|V,\left\langle\alpha, h^{\prime}\right\rangle\right| V$. Then $\alpha \mid V=\iota_{N} \circ \psi=\iota_{N} \circ \psi^{\prime}$ whence there is for each $g$ in $V$ an element $s[g]$ in $C$ satisfying $\psi^{\prime}(g)=s[g] \psi(g)$. It is now easy to check that if $g_{1}$, $g_{2}, g_{1} g_{2} \in V$, then

$$
h^{\prime}\left(g_{1}, g_{2}\right)=s\left[g_{1}\right]^{\alpha\left(g_{1}\right)} s\left[g_{2}\right] s^{-1}\left[g_{1} g_{2}\right] h\left(g_{1}, g_{2}\right)=\delta s\left[g_{1}, g_{2}\right] h\left(g_{1}, g_{2}\right) .
$$

Let us now enlarge $s: V \rightarrow C$ arbitrarily to a map $s: G \rightarrow C$ which we shall regard as an equivariant cochain on $\left(K_{G}\right)_{1}$. Let us further define

$$
h^{\prime \prime}\left(g_{1}, g_{2}\right)=\delta s\left[g_{1}, g_{2}\right] h\left(g_{1}, g_{2}\right)
$$

for all $g_{1}, g_{2}$ in $G$. Then $\left\langle\alpha, h^{\prime \prime}\right\rangle$ is admissible because $\left\langle\alpha, h^{\prime \prime}\right\rangle\left|V=\left\langle\alpha, h^{\prime}\right\rangle\right| V$. Moreover, with the aid of (8.1) we obtain $f_{\alpha, h^{\prime \prime}}=\delta(\delta s) \cdot f_{\alpha, h}=f_{\alpha, h}$. It will suffice to prove now that $f_{\alpha, h^{\prime}}\left(f_{\alpha, h^{\prime \prime}}\right)^{-1}$ is a coboundary.

From the premultiplicator property (7.1), applied to $\left\langle\alpha, h^{\prime}\right\rangle$ and $\left\langle\alpha, h^{\prime \prime}\right\rangle$, it follows that for every $g_{1}, g_{2} \in G$ there exists an $c\left[g_{1}, g_{2}\right] \in C$ such that

$$
h^{\prime}\left(g_{1}, g_{2}\right)=c\left[g_{1}, g_{2}\right] h^{\prime \prime}\left(g_{1}, g_{2}\right) \text {, }
$$

and as $h^{\prime}\left(g_{1}, g_{2}\right)=h^{\prime \prime}\left(g_{1}, g_{2}\right)$ when $g_{1}, g_{2}, g_{1} g_{2} \in V, c$ is an equivariant cochain on $K_{G}^{(V)}$. (8.2) implies $f_{\alpha, h^{\prime}}=\delta c \cdot f_{\alpha, h^{\mu}}$.

Proof of II. The required $\left\langle\alpha^{\prime}, h^{\prime}\right\rangle$ is given by Lemma 3 of $\S 7$, p. 304 .

Definition. Obs? $\langle G, N, \Theta\rangle=\left\{f_{\alpha, h}\right\} \in H^{3}(G \bmod V, C)$.

Suppose there exists an enlargement \&S of $\mathfrak{\&}$ with kernel $\langle G, N, \Theta\rangle$. Then we can choose for the admissible $\langle\alpha, h\rangle$ any multiplicator of (s) so that $f_{\alpha, h}=1$ and $\mathrm{Obs}_{\mathfrak{L}}\langle G, N, \Theta\rangle=0$.

Assume $\operatorname{Obs}_{\mathfrak{R}}\langle G, N, \Theta\rangle=0$. Taking any admissible $\langle\alpha, h\rangle$, we have $f_{\alpha, h}=\delta c$ for some $C$-valued cochain $c$ on $K_{G}$ which satisfies $c\left[g_{1}, g_{2}\right]=1$ whenever $g_{1}, g_{2}$, $g_{1} g_{2} \in V$. Let us define $h^{\prime}=c^{-1} \cdot h$. Then $\left\langle\alpha, h^{\prime}\right\rangle$ is again admissible, moreover $f_{\alpha, h^{\prime}}=(\delta c)^{-1} f_{\alpha, h}=1$. Hence $\left\langle\alpha, h^{\prime}\right\rangle$ is a multiplicator and it defines a global extension (\$). Since $\left\langle\alpha, h^{\prime}\right\rangle \mid V$ defines an extension isomorphic to $\mathbb{2}$ (see (a) in $\S 7$, p. 304), (S) is an enlargement of $\mathfrak{R}$. Since $\left\langle\alpha, h^{\prime}\right\rangle$ is a multiplicator of $(S)$ (see (b) in $\S 7)$, the kernel of $\mathbb{S}$ coincides with the kernel of $\left\langle\alpha, h^{\prime}\right\rangle$, that is with $\langle G, N, \Theta\rangle$. This proves the first part of the theorem.

Suppose now that $C$ is an arbitrary $G$-module and that $a \in H^{3}(G \bmod V, C)$ 
is given. To find $\mathfrak{L}$ and $\langle G, N, \Theta\rangle$, assume that $a$ is represented by a cocycle $f$ on $\left(K_{G}\right)_{3}$ satisfying $f \mid\left(K_{G}^{V}\right)_{3}=1$. It is shown in [3, Lemma 9.1, p. 334] that the cohomology class of $f$ in $H_{\mathrm{eq}}^{3}\left(K_{G}, C\right)=H^{3}(G, C)$ is the obstruction of some abstract kernel $\langle G, N, \Theta\rangle$ (in the sense of Lemma 2 in $\$ 7$, p. 304). More precisely, the proof of [3] yields for every $G$-module $C$ and for every $C$-valued equivariant cocycle $f$ on $\left(K_{G}\right)_{3}$, a group $N$ whose centre is $C$ and a premultiplicator $\langle\alpha, h\rangle$ from $G$ to $N$ which induces on $C$ the proper operators and satisfies $f_{\alpha, h}=f$. Since in our case $f_{\alpha, h}\left|\left(K_{G}^{v}\right)_{3}=1,\langle\alpha, h\rangle\right| V$ is a multiplicator from $V$ to $N$ and therefore defines a local extension $\mathfrak{L}$. If $\langle G, N, \Theta\rangle$ is the kernel of $\langle\alpha, h\rangle$, then $\langle G, N, \Theta\rangle$ is an enlargement of the kernel of $\langle\alpha, h\rangle \mid V$, i.e. of the kernel of $\mathfrak{L}$. It is obvious that $\langle\alpha, h\rangle$ is admissible for $\mathfrak{L}$ and $\langle G, N, \Theta\rangle$, whence $\operatorname{Obs} \mathfrak{R}\langle G, N, \Theta\rangle=\left\{f_{\alpha, h}\right\}=\{f\}=a$. This completes the proof of the theorem.

Let us show that $\operatorname{Obs} \_\langle G, N, \Theta\rangle$ behaves contravariantly under morphisms of extensions. More precisely, let $\hat{V}, V$ be local groups contained in the groups $\hat{G}, G$; let $\omega:(\hat{V}, \hat{G}) \rightarrow(V, G)$ be a morphism and let

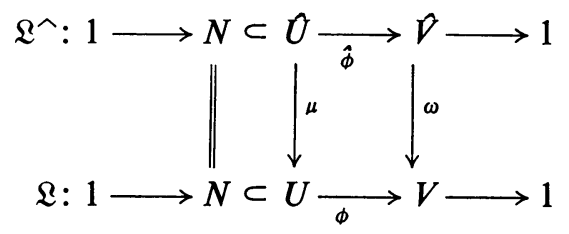

be a morphism of two local extensions $\mathfrak{L}^{\wedge}, \mathfrak{L}$. The above diagram implies that, if we choose a $\phi$-selector $\psi$, then there exists a $\hat{\psi}$-selector $\hat{\psi}$ satisfying

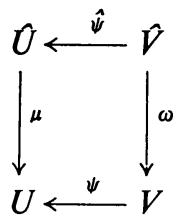

Let $\left\langle V, N, \Theta_{V}\right\rangle$ be the abstract kernel of $\mathfrak{R}$. We assert that $\left\langle\hat{V}, N, \Theta_{V} \circ \omega\right\rangle$ is the kernel of $\mathfrak{L}^{\wedge}$. Indeed, this follows from the equality

$$
\begin{aligned}
& \hat{V} \underset{\omega}{\longrightarrow} V \underset{\psi}{\longrightarrow} U \underset{\iota_{N}}{\longrightarrow} A(N) \\
& \quad=\hat{V} \underset{\hat{\psi}}{\longrightarrow} \hat{U} \underset{\mu}{\longrightarrow} U \underset{\iota_{N}}{\longrightarrow} A(N)=\hat{V} \underset{\hat{\psi}}{\longrightarrow} \hat{U} \underset{\hat{\imath}_{N}}{\longrightarrow} A(N)
\end{aligned}
$$

which is a consequence of (8.3). Now let $\langle G, N, \Theta\rangle$ be an enlargement of the kernel of $\mathfrak{L}$ and let $C$ (the centre of $N$ ) have the induced $G$-structure. It follows that $\langle\hat{G}, N, \Theta \circ \omega\rangle$ is an enlargement of the kernel of $\mathfrak{L}^{\wedge}$, and $\left.\operatorname{Obs}_{\mathfrak{R}} \wedge \hat{G}, N, \Theta \circ \omega\right\rangle$ $\in H^{3}(\hat{G} \bmod \hat{V}, C)$, where the $\hat{G}$-structure of $C$ is induced by $\langle\hat{G}, N, \Theta \circ \omega\rangle$, hence is derived from the $G$-structure by the change of groups $\omega: \hat{G} \rightarrow G$ (see $\S 6$, p. 301$)$. As in $\S 6, \omega:(\hat{V}, \hat{G}) \rightarrow(V, G)$ induces

$$
\omega_{\mathrm{eq}}^{3}: H^{3}(G \bmod V, C) \rightarrow H^{3}(\hat{G} \bmod \hat{\nabla}, C) .
$$

LemMA. Obs $\mathfrak{R}\langle\hat{G}, N, \Theta \circ \omega\rangle=\omega_{\text {eq }}^{3}\left(\mathrm{Obs}_{\mathfrak{L}}\langle G, N, \Theta\rangle\right)$. 
Proof. Let $\langle\alpha, h\rangle$ be a premultiplicator from $G$ to $N$ which is admissible for $\mathfrak{R}$, $\langle G, N, \Theta\rangle$. Suppose the restriction $\langle\alpha, h\rangle \mid V$ is the multiplicator defined by a $\phi$-selector $\psi$; we denote by $\hat{\psi}$ a $\hat{\phi}$-selector satisfying (8.3). Let us prove that $\langle\alpha \circ \omega, h \circ(\omega \times \omega)\rangle$ is admissible for $\mathfrak{R}^{\wedge},\langle\hat{G}, N, \Theta \circ \omega\rangle$. That this is a premultiplicator for $\langle\hat{G}, N, \Theta \circ \omega\rangle$ follows from

$$
\Theta \circ \omega: \hat{G} \underset{\omega}{\longrightarrow} G \underset{\alpha}{\longrightarrow} A(N) \longrightarrow A(N) / I(N) .
$$

We assert that $\langle\alpha \circ \omega, h \circ(\omega \times \omega)\rangle \mid \hat{V}$ is the multiplicator defined by $\mathfrak{Q}^{\wedge}$ and $\hat{\psi}$. Indeed, $\alpha \circ \omega \mid \hat{V}=\hat{\iota}_{N} \circ \hat{\psi}$ by (8.4). Moreover, if for given $g_{1}, g_{2}, g_{1} g_{2} \in \hat{V}, \hat{h}\left(g_{1}, g_{2}\right)$ denotes the element of $N$ which satisfies

$$
\hat{\psi}\left(g_{1}\right) \hat{\psi}\left(g_{2}\right)=\hat{h}\left(g_{1}, g_{2}\right) \hat{\psi}\left(g_{1} g_{2}\right)
$$

then, by application of $\mu$ and using (8.3),

$$
\psi\left(\omega g_{1}\right) \psi\left(\omega g_{2}\right)=\hat{h}\left(g_{1}, g_{2}\right) \psi\left(\omega\left(g_{1} g_{2}\right)\right) .
$$

Since the same equation is satisfied by $h\left(\omega g_{1}, \omega g_{2}\right)$ at place of $\hat{h}\left(g_{1}, g_{2}\right)$, we have $\hat{h}=h \circ(\omega \times \omega)$. Clearly

$$
f_{\alpha \circ \omega, h_{\circ}(\omega \times \omega)}\left[g_{1}, g_{2}, g_{3}\right]=f_{\alpha, h}\left[\omega g_{1}, \omega g_{2}, \omega g_{3}\right],
$$

so that the cocycle $f_{\alpha \circ \omega, h_{\circ}(\omega \times \omega)}$ is obtained from $f_{\alpha, h}$ by superposition with the map of chain complexes $K_{G \wedge}^{(V \wedge)} \rightarrow K_{G}^{(V)}$ induced by $\omega:(\hat{V}, \hat{G}) \rightarrow(V, G)$, (see $\S 6$, p. 302). Hence

$$
\operatorname{Obs}_{\mathfrak{R}}\langle\hat{G}, N, \Theta \circ \omega\rangle=\left\{f_{\alpha_{\bullet} \omega, h \circ(\omega \times \omega)}\right\}=\omega_{\text {eq }}^{3}\left\{f_{\alpha, h}\right\}=\omega_{\text {eq }}^{3} \operatorname{Obs}_{\mathfrak{R}}\langle G, N, \Theta\rangle .
$$

9. The difference classes $d_{\mathfrak{l}}\left(\mathscr{S}_{0}, \mathscr{S}_{1}\right), d\left(\mathfrak{Q}_{0}, \mathfrak{Q}_{1}\right)$. Let $\mathfrak{L}: 1 \rightarrow N \subset U \rightarrow_{\phi} V \rightarrow 1$ be a local extension which is enlargeable over $G$. Then its local kernel is enlargeable, say to $\langle G, N, \Theta\rangle$. We endow the centre $C$ of $N$ with the $G$-structure induced by $\langle G, N, \Theta\rangle$.

LEMMA 1. To any two enlargements $\mathbb{H}_{i}: 1 \rightarrow N \subset H_{i} \rightarrow_{\phi_{i}} G \rightarrow 1(i=0,1)$ of $\mathfrak{L}$ which have the kernel $\langle G, N, \Theta\rangle$ corresponds an element $d_{\mathfrak{Q}}\left(\mathscr{S}_{0}, \mathbb{S}_{1}\right)$ of

such that

$$
H^{2}(G \bmod V, C)
$$

(i) $d_{\mathfrak{R}}\left(\mathscr{S}_{1}, \mathscr{S}_{2}\right)-d_{\mathfrak{L}}\left(\mathscr{S}_{0}, \mathscr{S}_{2}\right)+d_{\mathfrak{L}}\left(\mathscr{S}_{0}, \mathscr{S}_{1}\right)=0$,

(ii) $d_{\mathfrak{Q}}\left(\mathscr{S}_{0}, \mathscr{S}_{1}\right)=0$ iff $\mathscr{S}_{0}$ and $\mathscr{S}_{1}$ are isomorphic over $\mathfrak{L}$,

(iii) for every enlargement $\mathfrak{S}_{0}$ with kernel $\langle G, N, \Theta\rangle$ and every $d \in$ $H^{2}(G \bmod V, C)$, there exists an enlargement $\mathfrak{S}_{1}$ such that $d_{\mathfrak{L}}\left(\mathfrak{S}_{0}, \mathfrak{S}_{1}\right)=d$.

Combining the above properties (i), (ii), (iii) and using the theorem of $\S 8$, p. 305 we derive the following

COROLlaRY 1. If $\mathrm{Obs}_{\mathfrak{R}}\langle G, N, \Theta\rangle=0$, then the isomorphism classes over $\mathfrak{Q}$ of enlargements of $\mathfrak{Q}$ with abstract kernel $\langle G, N, \Theta\rangle$ can be put in $1: 1$ correspondence with the elements of $H^{2}(G \bmod V, C)$. 
COROLlary 2. If $\mathfrak{L}$ is enlargeable over $G$ and $V$ generates $G$, then the isomorphism classes over $\mathfrak{L}$ of enlargements over $G$ of $\mathfrak{L}$ can be put in $1: 1$ correspondence with the elements of $\operatorname{Hom}_{G}\left(H_{1}\left(\Gamma_{G}^{V}\right), C\right)$.

Indeed, if $V$ generates $G$, the abstract kernel of $\mathfrak{L}$ has a unique enlargement over $G$, so that the $G$-structure of $C$ is well determined. The conclusion follows now from Lemma 2 in $\S 6$ (p. 301), Corollary 1 above, and the theorem in $\S 8$ (p. 305). If $\Gamma_{G}^{V}$ is acyclic in dimension 1 (and this is always the case when $G$ is $V$-monodrome, by Lemma $2, \S 3$, p. 296), then all enlargements over $G$ of $\mathfrak{L}$ are isomorphic.

To define $d_{\mathfrak{L}}\left(\mathscr{S}_{0}, \mathbb{S}_{1}\right)$, let us call a triple $\left\langle\alpha, h_{0}, h_{1}\right\rangle$ admissible if $\left\langle\alpha, h_{i}\right\rangle$ are multiplicators of $\mathscr{B S}_{\mathfrak{i}}$ such that $\left\langle\alpha, h_{\mathfrak{i}}\right\rangle \mid V$, (which then are multiplicators of $\mathfrak{L}$ ), are defined by the same $\phi$-selector $\psi: V \rightarrow U$. The existence is derived from Lemma 3 in $\S 7$, p. 304. Indeed we always can choose $\phi_{i}$-selectors $\psi_{i}$ such that $\psi_{0}=\psi_{1}$ on $V$, these will define multiplicators $\left\langle\alpha_{i}, h_{i}\right\rangle$ such that $\left\langle\alpha_{i}, h_{i}\right\rangle \mid V$ are given by the same $\phi$-selector. Then $\alpha_{0}=\alpha_{1}$ on $V$. Also, $\left\langle\alpha_{i}, h_{i}\right\rangle$ have the same kernel, hence $\alpha_{0}$ factorizes the kernel of $\left\langle\alpha_{1}, h_{1}\right\rangle$. It follows from Lemma $3, \S 7$ that there is another multiplicator $\left\langle\alpha_{0}, h_{1}^{\prime}\right\rangle$ for $\mathscr{S S}_{1}$ with the property that $\left\langle\alpha_{1}, h_{1}\right\rangle\left|V,\left\langle\alpha_{0}, h_{1}^{\prime}\right\rangle\right| V$ are defined by the same $\phi$-selector. Thus $\left\langle\alpha_{0}, h_{0}\right\rangle\left|V,\left\langle\alpha_{0}, h_{1}^{\prime}\right\rangle\right| V$ are defined by the same $\phi$-selector.

If $\left\langle\alpha, h_{0}, h_{1}\right\rangle$ is admissible and $\psi_{i}$ denotes the $\phi_{i}$-selector defining $\left\langle\alpha, h_{i}\right\rangle$, then we have

$$
\psi_{i}\left(g_{1}\right) \psi_{i}\left(g_{2}\right)=h_{i}\left(g_{1}, g_{2}\right) \psi_{i}\left(g_{1} g_{2}\right) \text { for all } g_{1}, g_{2} \in G,
$$

and $\alpha=\iota_{N} \circ \psi_{i}$. These equalities imply the existence of an equivariant $C$-valued cochain $c_{\alpha, h_{0}, h_{1}}$ on $\left(K_{G}\right)_{2}$ such that

$$
h_{1}\left(g_{1}, g_{2}\right)=c_{\alpha, h_{0}, h_{1}}\left[g_{1}, g_{2}\right] h_{0}\left(g_{1}, g_{2}\right) \text { for all } g_{1}, g_{2} \in G \text {. }
$$

Since $c_{\alpha, h_{0}, h_{1}} \mid\left(K_{G}^{V}\right)_{2}=1$, we can regard $c_{\alpha, h_{0}, h_{1}}$ as a cochain on $K_{G}^{(V)}$.

Putting $h_{1}=c_{\alpha, h_{0}, h_{1}} \cdot h_{0}$ in $f_{\alpha, h_{1}}$, we obtain $f_{\alpha, h_{1}}=\delta c_{\alpha, h, h_{0}} \cdot f_{\alpha, h_{0}}$ and since $f_{\alpha, h_{t}}=1$, it follows that $c_{\alpha, h_{0}, h_{1}}$ is a cocycle. Its cohomology class does not depend on the admissible triple $\left\langle\alpha, h_{0}, h_{1}\right\rangle$. To show this, it is enough to verify that

I. If $\left\langle\alpha, h_{0}, h_{1}\right\rangle,\left\langle\alpha, h_{0}^{\prime}, h_{1}^{\prime}\right\rangle$ are admissible, then there exists an equivariant cochain $c$ on $K_{G}^{(V)}$ such that

$$
c_{\alpha, h_{0}^{\prime}, h_{1}^{\prime}}\left(c_{\alpha, h_{0}, h_{1}}\right)^{-1}=\delta c .
$$

II. If $\left\langle\alpha, h_{0}, h_{1}\right\rangle$ and $\left\langle\alpha^{\prime}, h_{0}^{\prime \prime}, h_{1}^{\prime \prime}\right\rangle$ are admissible, then there exists an admissible $\left\langle\alpha^{\prime}, h_{0}^{\prime}, h_{1}^{\prime}\right\rangle$ such that

$$
c_{\alpha, h_{0}, h_{1}}=c_{\alpha^{\prime}, h_{0}^{\prime}, h_{1}^{\prime}} .
$$

Proof of I. Let $\psi_{i}$ be the $\phi_{i}$-selectors defining $\left\langle\alpha, h_{i}\right\rangle$, and let $\psi_{i}^{\prime}$ be similarly defined for $\left\langle\alpha, h_{i}^{\prime}\right\rangle$. We have $\alpha=\iota_{N} \circ \psi_{i}=\iota_{N} \circ \psi_{i}^{\prime}$, whence there is a $C$-valued cochain $c_{i}$ on $\left(K_{G}\right)_{1}$ such that $\psi_{i}^{\prime}=c_{i} \cdot \psi_{i}$. This implies by easy computation that $h_{i}^{\prime}=\delta c_{i} \cdot h_{i}$. Hence

$$
c_{\alpha, h_{0}^{\prime}, h_{1}^{\prime}}=h_{1}^{\prime}\left(h_{0}^{\prime}\right)^{-1}=\delta c_{1} \cdot \delta c_{0}^{-1} \cdot h_{1} \cdot h_{0}^{-1}=\delta\left(c_{1} c_{0}^{-1}\right) c_{\alpha, h_{0}, h_{1}}
$$


We define $c=c_{1} c_{0}^{-1}$. Then $c=1$ on $V$ since $\psi_{0}=\psi_{1}$ and $\psi_{0}^{\prime}=\psi_{1}^{\prime}$ on $V$, and we can regard $c$ as a cochain on $K_{G}^{(V)}$. This proves $\mathrm{I}$.

REMARK. We deduce from the above proof that if $\left\langle\alpha, h_{0}, h_{1}\right\rangle$ is admissible and $c$ is an arbitrary $C$-valued cochain on $\left(K_{G}^{(V)}\right)_{1}$, then there exists an admissible $\left\langle\alpha, h_{0}^{\prime}, h_{1}^{\prime}\right\rangle$ satisfying the equality in I. Indeed, if $\psi_{i}$ are the $\phi_{i}$-selectors defining $\left\langle\alpha, h_{i}\right\rangle$, then let us set $c_{0}=1, c_{1}=c$ and $\psi_{i}^{\prime}=c_{i} \psi_{i}$. Then the $\phi_{i}$-selectors $\psi_{i}^{\prime}$ define an admissible triple $\left\langle\alpha, h_{0}^{\prime}, h_{1}^{\prime}\right\rangle$ and it follows as in the preceding proof that

$$
c_{\alpha, h_{0}^{\prime}, h_{1}^{\prime}}\left(c_{\alpha, h_{0}, h_{1}}\right)^{-1}=\delta c .
$$

Proof of II. Since $\alpha^{\prime}$ factorizes the kernel of $\left\langle\alpha, h_{i}\right\rangle$, we can apply Lemma 3 of $\S 7$, p. 304 , to find a multiplicator $\left\langle\alpha^{\prime}, h_{i}^{\prime}\right\rangle$ for $\mathscr{S}_{i}$. It follows from the proof of the lemma that $\left\langle\alpha^{\prime}, h_{i}^{\prime}\right\rangle$ is defined by $\psi_{i}^{\prime}=k \cdot \psi_{i}$ where $k$ depends only on $\alpha, \alpha^{\prime}$. Therefore $\psi_{0}^{\prime}=\psi_{1}^{\prime}$ on $V$ and $\left\langle\alpha^{\prime}, h_{0}^{\prime}, h_{1}^{\prime}\right\rangle$ is admissible. Looking again at the proof of Lemma 3 in $\$ 7$ we find that

$$
h_{i}^{\prime}\left(g_{1}, g_{2}\right)=k\left(g_{1}\right)^{\alpha\left(g_{1}\right)} k\left(g_{2}\right) h_{i}\left(g_{1}, g_{2}\right) k^{-1}\left(g_{1} g_{2}\right) .
$$

Since $h_{1} h_{0}^{-1}=c_{\alpha, h_{0}, h_{1}}, h_{1}^{\prime}\left(h_{0}^{\prime}\right)^{-1}=c_{\alpha^{\prime}, h_{0}^{\prime}, h_{1}^{\prime}}$ are in the centre of $N$, equations (9.1) imply that they are equal.

We define now

$$
d_{\mathfrak{L}}\left(\mathscr{S}_{0}, \mathscr{S S}_{1}\right)=\left\{c_{\alpha, h_{0}, h_{1}}\right\} \in H^{2}(G \bmod V, C) .
$$

Property (i) is easily established by considering three multiplicators $\left\langle\alpha, h_{i}\right\rangle$ whose restrictions to $V$ are defined by the same $\phi$-selector $\psi$. To show (ii) note that if $\mathfrak{S}_{0}, \mathfrak{S}_{1}$ are isomorphic over $\mathfrak{L}$, then they have a common multiplicator $\langle\alpha, h\rangle$ (we have to choose $\phi_{i}$-selectors which commute with the isomorphism). Hence $d_{\mathfrak{Q}}\left(\mathscr{S}_{0}, \mathscr{S S}_{1}\right)=\left\{c_{\alpha, h, h}\right\}=0$. Conversely, if $d_{\mathfrak{L}}\left(\mathscr{S}_{0}, \mathfrak{S S}_{1}\right)=0$ then, by the remark after the proof of I, we can assume that $c_{\alpha, h_{0}, h_{1}}=1$ holds for some admissible $\left\langle\alpha, h_{0}, h_{1}\right\rangle$. That is, $\mathbb{S}_{0}, \mathbb{S}_{1}$ have a common multiplicator defined by $\phi_{i}$-selectors which coincide on $V$, and hence $\mathfrak{S}_{0}, \mathfrak{S}_{1}$ must be isomorphic over $\mathfrak{L}$ (see (a) in $\S 7$, p. 304).

To prove (iii) we take a $\phi_{0}$-selector $\psi_{0}$, denote by $\left\langle\alpha, h_{0}\right\rangle$ the corresponding multiplicator and denote by $c$ a cocycle on $K_{G}$ representing the given element $d \in H^{2}(G \bmod V, C)$. We define

$$
h_{1}\left(g_{1}, g_{2}\right)=c\left[g_{1}, g_{2}\right] h_{0}\left(g_{1}, g_{2}\right) \text { for all } g_{1}, g_{2} \text { in } G \text {. }
$$

Then $\left\langle\alpha, h_{1}\right\rangle$ is again a multiplicator since $h_{1}[1,1]=1$ by $c \mid\left(K_{G}^{V}\right)_{2}=1$ and $f_{\alpha, h_{1}}=\delta c \cdot f_{\alpha, h}=1$. Let $\mathfrak{S}_{1}$ be the corresponding extension. The elements of $H_{1}$ are pairs $\langle n, g\rangle(n \in N, g \in G)$, and the equality $h_{1}\left(g_{1}, g_{2}\right)=h_{0}\left(g_{1}, g_{2}\right)$ for $g_{1}, g_{2}$, $g_{1} g_{2} \in V$ implies that the map $U \rightarrow H_{1}$ given by $n \psi_{0}(g) \rightarrow\langle n, g\rangle$ is an isomorphism of $U$ onto $N \times V \subset H_{1}$. We use it to identify $U$ with $N \times V$. Then $\mathscr{S}_{1}$ becomes an enlargement of $\mathfrak{L}$. Moreover the $\phi_{1}$-selector $\psi_{1}(g)=\langle 1, g\rangle$ defines the multiplicator $\left\langle\alpha, h_{1}\right\rangle$ and $\psi_{1}\left|V=\psi_{0}\right| V$ under the identification. Hence $\left\langle\alpha, h_{0}, h_{1}\right\rangle$ is admissible and (9.2) shows that $d_{\mathfrak{R}}\left(\mathscr{S S}_{0}, \mathscr{S}_{1}\right)=\{c\}=d$. This completes the proof of Lemma 1 .

Let $\langle V, N, \Theta\rangle$ be an abstract kernel and let $C=$ centre of $N$ be endowed with the $V$-module structure induced by $\langle V, N, \Theta\rangle$. 
Lemma 2. To any two local extensions $\mathfrak{L}_{i}: 1 \rightarrow N \subset U_{i} \rightarrow_{\phi_{i}} V \rightarrow 1,(i=0,1)$, which have the kernel $\langle V, N, \Theta\rangle$ corresponds an element $d\left(\mathfrak{Q}_{0}, \mathfrak{\Omega}_{1}\right) \in H^{2}(V, C)$ such that

(i) $d\left(\mathfrak{Q}_{1}, \mathfrak{\Omega}_{2}\right)-d\left(\mathfrak{\Omega}_{0}, \mathfrak{\Omega}_{2}\right)+d\left(\mathfrak{\Omega}_{0}, \mathfrak{L}_{1}\right)=0$,

(ii) $d\left(\mathfrak{Q}_{0}, \mathfrak{Q}_{1}\right)=0$ iff there exists an isomorphism between $\mathfrak{L}_{0}, \mathfrak{L}_{1}$ which on $N$ is the identity,

(iii) for every extension $\mathfrak{\Omega}_{0}$ with kernel $\langle V, N, \Theta\rangle$ and for every $d \in H^{2}(V, C)$, there exists an extension $\mathfrak{L}_{1}$ such that $d\left(\mathfrak{Q}_{0}, \mathfrak{L}_{1}\right)=d$.

Corollary. If Obs $\langle V, N, \Theta\rangle=0$, then the isomorphism classes of extensions with kernel $\langle V, N, \Theta\rangle$ can be put in $1: 1$ correspondence with the elements of $H^{2}(V, C)$.

This follows by applying Lemma 2 of $\S 7$, p. 304. The above Lemma 2 is a special case of the preceding Lemma 1 , obtained by substituting $V$ and 1 for $G$ and $V$ respectively. An inspection of the proof shows that we had no need to assume that $G$ is a group, a local group would have done as well. All steps of the proof remain valid after this substitition except the part of the proof of (iii) which uses the fact that a cochain $c$ on $\left(K_{G}^{(V)}\right)_{2}$ satisfies $c[1,1]=1$. This is not true for a 2-dimensional $V$-cochain, but we can always alter a $V$-cochain by a $V$-coboundary, so as to achieve the equality (see proof of Lemma 2 in $\$ 7$, p. 304).

Let us establish now a connection between obstructions and difference classes of extensions. Let

$$
\mathfrak{L}_{i}: 1 \longrightarrow N \subset U_{i} \underset{\phi_{i}}{\longrightarrow} V \longrightarrow 1, i=0,1,
$$

where $V$ is contained in a group $G$. We assume that the kernel of $\mathfrak{L}_{i}$ is enlargeable to some abstract kernel $\langle G, N, \Theta\rangle$ and we take $C$ with the $G$-structure induced by the latter. Let

$$
\partial_{E}^{\mathrm{eq}}: H^{2}(V, C) \rightarrow H^{3}(G \bmod V, C)
$$

be the connecting homomorphism in the exact cohomology sequence of the exact sequence $E=E_{G}^{V}($ see $\S 6$, p. 300$)$.

LemmA 3. $\partial_{E}^{\mathrm{eq}}\left(d\left(\mathfrak{Q}_{0}, \mathfrak{\Omega}_{1}\right)\right)=\mathrm{Obs}_{\mathfrak{R}_{1}}\langle G, N, \Theta\rangle-\mathrm{Obs}_{\mathfrak{R}_{0}}\langle G, N, \Theta\rangle$.

Proof. Let $\langle\alpha, h\rangle$ be a premultiplicator of $\langle G, N, \Theta\rangle$. Then $\alpha$ factorizes $\langle G, N, \Theta\rangle$ and hence by Lemma 3 of $\S 7$ (p. 304), we have for $i=0,1$ an $\left\langle\alpha, h_{i}\right\rangle$ admissible for $\mathfrak{L}_{i},\langle G, N, \Theta\rangle$ in the sense of $\S 8$, and such that $d\left(\mathfrak{L}_{0}, \mathfrak{L}_{1}\right)$ is represented by the equivariant cocycle $c_{\alpha, h_{0}, h_{1}}$ on $\left(K_{G}^{V}\right)_{2}$ satisfying

$$
h_{1}\left(g_{1}, g_{2}\right)=c_{\alpha, h_{0}, h_{1}}\left[g_{1}, g_{2}\right] h_{0}\left(g_{1}, g_{2}\right) \text { whenever } g_{1}, g_{2}, g_{1} g_{2} \in V \text {. }
$$

Let us define a cocycle $\bar{c}_{\alpha, h_{0}, h_{1}}$ on $\left(K_{G}\right)_{2}$ by

$$
\begin{aligned}
\bar{c}_{\alpha, h_{0}, h_{1}}\left[g_{1}, g_{2}\right] & =c_{\alpha, h_{0}, h_{1}}\left[g_{1}, g_{2}\right] \text { if } g_{1}, g_{2}, g_{1} g_{2} \in V, \\
& =1 \text { if otherwise. }
\end{aligned}
$$


Let us further define

$$
\begin{aligned}
\bar{h}_{i}\left(g_{1}, g_{2}\right) & =h_{i}\left(g_{1}, g_{2}\right) \text { if } g_{1}, g_{2}, g_{1} g_{2} \in V, \\
& =h\left(g_{1}, g_{2}\right) \text { if otherwise. }
\end{aligned}
$$

Clearly $\left\langle\alpha, \bar{h}_{i}\right\rangle$ is admissible for $\mathfrak{E}_{i},\langle G, N, \Theta\rangle$. Moreover $\bar{h}_{1}=\bar{c}_{\alpha, h_{0}, h_{1}} \cdot \bar{h}_{0}$. This implies $f_{\alpha, h_{1}}=\delta \bar{c}_{\alpha, h_{0}, h_{1}} \cdot f_{\alpha, h_{0}}$, and the result follows by passing to cohomology classes.

10. The enlargeability of extensions. Let $\mathfrak{L}: 1 \rightarrow N \subset U \rightarrow_{\phi} V \rightarrow 1$ be a local extension and let $G$ be a group containing $V$. We assume in this section that $G$ is generated by $V$. If there exists an enlargement (SS of $\mathfrak{L}$ over $G$, then it is clear that the abstract kernel of $\mathfrak{L}$ is enlargeable over $G$. Thus, trying to enlarge $\mathfrak{L}$ over $G$, we may try first to enlarge the kernel $\left\langle V, N, \Theta_{V}\right\rangle$ of $\mathfrak{L}$ to an abstract kernel $\langle G, N, \Theta\rangle$; the latter, if it exists, will be unique since $V$ generates $G$. As we have shown in Lemma 1 of $\S 3$ (p. 295), this can be done iff $\operatorname{Obs}\left(\Theta_{V}, G\right)$ vanishes. We define

$$
\operatorname{Obs}_{1}(\mathfrak{L}, G)=\operatorname{Obs}\left(\Theta_{V}, G\right): \pi_{1}\left(\Gamma_{G}^{V}\right) \rightarrow A(N) / I(N)
$$

calling this the first obstruction of $\mathfrak{Q}$ over $G$.

Suppose that $\mathrm{Obs}_{1}(\mathfrak{L}, G)$ vanishes, denote by $\langle G, N, \Theta\rangle$ the unique enlargement of the kernel of $\mathfrak{L}$, and consider $C$ (=centre of $N$ ) with the induced $G$-module structure. We define

$$
\mathrm{Obs}_{2}(\mathfrak{L}, G)=\mathrm{Obs}_{\mathfrak{L}}\langle G, N, \Theta\rangle \in H^{3}(G \bmod V, C)
$$

calling this the second obstruction of $\mathfrak{Q}$ over $G$. From the theorem in $\S 8$ it follows now that $\mathfrak{R}$ is enlargeable over $G$ iff $\operatorname{Obs}_{2}(\mathfrak{L}, G)=0$. There may be many nonisomorphic enlargements and their isomorphism classes over $\mathfrak{L}$ can be put in $1: 1$ correspondence with the elements of $\operatorname{Hom}_{G}\left(H_{1}\left(\Gamma_{G}^{V}\right), C\right)$ (Corollary 2 in $\S 9$, p. 309).

TheOREM. Let $\omega:(\hat{V}, \hat{G}) \rightarrow(V, G)$ be a morphism, where the groups $G, \hat{G}$ are generated by $V, \hat{V}$; let $\mathfrak{Q}^{\wedge} \rightarrow \mathfrak{L}$ be a morphism of extensions

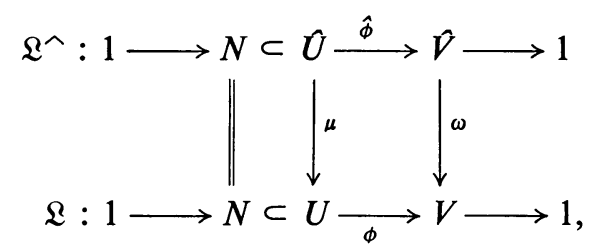

let $\omega_{\mathrm{eq}}^{3}: H^{3}(G \bmod V, C) \rightarrow H^{3}(\hat{G} \bmod \hat{V}, C)$ be the morphism defined in $\S 6$, p. 302 and let $\omega_{\pi}: \pi_{1}\left(\Gamma_{G^{\wedge}}^{V \wedge} \rightarrow \pi_{1}\left(\Gamma_{G}^{V}\right)\right.$ be the morphism defined in $\S 3, p .296$. Then

$$
\operatorname{Obs}_{1}\left(\mathfrak{L}^{\wedge}, \hat{G}\right)=\operatorname{Obs}_{1}(\mathfrak{R}, G) \circ \omega_{\pi},
$$

and if $\mathrm{Obs}_{1}\left(\mathfrak{L}^{\wedge}, \hat{G}\right)$ vanishes, then

$$
\mathrm{Obs}_{2}\left(\mathfrak{Q}^{\wedge}, \hat{G}\right)=\omega_{\mathrm{eq}}^{3}\left(\mathrm{Obs}_{2}(\mathfrak{L}, G)\right) .
$$


Proof. The statement about $\mathrm{Obs}_{1}$ follows from the observation made in $\S 8$ that $\langle\hat{V}, N, \Theta \circ \omega\rangle$ is the kernel of $\mathfrak{L}^{\wedge}$ (see (8.4), p. 307) and from Lemma 3 in $\S 3$, p. 296. The part about $\mathrm{Obs}_{2}$ is a consequence of the lemma in $\S 8$, p. 307.

In the following four corollaries we identify $H_{i}\left(\Gamma_{G}^{V}\right)$ with $H_{i}\left(K_{G}^{V}\right)$ by using the canonical isomorphism between these groups resulting from the isomorphism of chain complexes $\sigma: \mathbb{\mho}\left(\Gamma_{G}^{V}\right) \rightarrow K_{G}^{V}$ (see $\S 4$, p. 297).

COROLlaRY 1. Supposing, in addition to the assumptions of the theorem, that

(i) $\operatorname{Obs}_{1}\left(\mathfrak{Q}^{\wedge}, \hat{G}\right)$ vanishes, (e.g. when $\hat{G}$ is $\hat{V}$-monodrome),

(ii) $H_{1}\left(\Gamma_{G \wedge}^{V \wedge}\right)=0$ (e.g. when $\hat{G}$ is $\hat{\nabla}$-monodrome, Lemma 2 of $\left.\S 3\right)$,

(iii) $\omega_{2}: H_{2}\left(\Gamma_{G \wedge}^{V \wedge}\right) \rightarrow H_{2}\left(\Gamma_{G}^{V}\right)$ is trivial (see $\left.\S 6, p .302\right)$,

we have that $\mathfrak{L}^{\wedge}$ is enlargeable over $\hat{G}$ and the enlargement is unique.

Proof. The uniqueness follows from (ii) by Corollary 2 to the lemma in $\$ 9$, p. 309. The existence of an enlargement will be a consequence of the above theorem provided we show $\omega_{\text {eq }}^{3}=0$. To show this we use Lemma 3 in $\$ 6$ (p. 302), by which $\hat{\kappa} \circ \omega_{\text {eq }}^{3}=\bar{\omega}_{2} \circ \kappa$, and since $\bar{\omega}_{2}=0$ by (iii) above, and $\hat{\kappa}$ is an isomorphism by Lemma 2 in $\S 6$ (p. 301), we finally have $\omega_{\text {eq }}^{3}=0$.

Suppose we have a morphism of extensions $\mathfrak{L}^{\wedge} \rightarrow \mathfrak{L}$ (10.1) in which $\mu$ and $\omega$ are inclusions. Then $\hat{U}=\phi^{-1} \hat{\nabla}$ and the local group structure of $\theta$ is determined by $\hat{U} \subset U$, so $\mathfrak{L}^{\wedge}$ is completely defined by $\mathfrak{L}$ and the symmetric subset $\hat{V} \subset V$. We shall call $\mathfrak{L}^{\wedge}$ the subextension of $\mathfrak{L}$ over $\hat{V}$ and we shall say that $\mathfrak{L}$ is enlargeable over $\hat{G}$ from $\hat{V}$ if $\mathfrak{L}^{\wedge}$ is enlargeable over $\hat{G}$. Combining the above theorem and Corollary 1 , we obtain

Corollary 2. Let $\omega:(\hat{V}, \hat{G}) \rightarrow(V, G)$ be a morphism, where $\hat{G}, G$ are groups generated by $\hat{V}, V ; \hat{V} \subset V$ and $\omega \mid \hat{V}$ is the inclusion. Assume further that

(i) $\omega_{\pi}: \pi_{1}\left(\Gamma_{G \wedge}^{V \wedge}\right) \rightarrow \pi_{1}\left(\Gamma_{G}^{V}\right)$ is trivial,

(ii) $H_{1}\left(\Gamma_{G \wedge}^{V \wedge}\right)=0$,

(iii) $\omega_{2}: H_{2}\left(\Gamma_{G \wedge}^{V \wedge}\right) \rightarrow H_{2}\left(\Gamma_{G}^{V}\right)$ is trivial.

Then every local extension over $V$ is enlargeable over $\hat{G}$ from $\hat{\nabla}$.

A partial converse is given by the following

Corollary 3. Let $\omega:(\hat{V}, \hat{G}) \rightarrow(V, G)$ be as in Corollary 2. Assume further that (i) $H_{1}\left(\Gamma_{G}^{V}\right)=0$,

(ii) $\omega_{2}: H_{2}\left(\Gamma_{G \wedge}^{V \wedge}\right) \rightarrow H_{2}\left(\Gamma_{G}^{V}\right)$ is nontrivial.

Then there exists a local extension $\mathfrak{Q}$ over $V$ which is not enlargeable over $\hat{G}$ from $\hat{\nabla}$.

Proof. Consider for $n=3$ the isomorphism $\kappa$ given in Lemma 2 of $\$ 6$ (p. 301), where $C$ is the $G$-module $H_{2}\left(K_{G}^{V}\right)$. Let $a$ be that element of $H^{3}\left(G \bmod V, H_{2}\left(K_{G}^{V}\right)\right)$ whose image under $\kappa$

$$
\kappa a: H_{2}\left(K_{G}^{V}\right) \rightarrow H_{2}\left(K_{G}^{V}\right)
$$

is the identity map. By the theorem in $\S 8$, p. 305 , there exists an extension $\mathfrak{L}$ over $V$ such that $H_{2}\left(K_{G}^{V}\right)=$ centre $N$ and whose abstract kernel is enlargeable to a kernel $\langle G, N, \Theta\rangle$ for which $a=\operatorname{Obs}_{\mathfrak{R}}\langle G, N, \Theta\rangle$, i.e. $a=\mathrm{Obs}_{2}(\mathfrak{R}, G)$. Let $\mathfrak{L}^{\wedge}$ be the 
sub-extension over $\hat{\mathcal{V}}$, then we have (10.1) with inclusions $\mu, \omega$, and by the above theorem it will suffice to show that $\omega_{\text {eq }}^{3} a \neq 0$. But by Lemma 3 of $\S 6$ (p. 302)

$$
\hat{\kappa}\left(\omega_{\mathrm{eq}}^{3} a\right)=\bar{\omega}_{2}(\kappa a)=\bar{\omega}_{2}(i d)=\omega_{2} \neq 0,
$$

which completes the proof.

Putting together the two preceding corollaries, we obtain

Corollary 4. If $\omega:(\hat{V}, \hat{G}) \rightarrow(V, G)$ is as in Corollary 2 and

(i) $\omega_{\pi}: \pi_{1}\left(\Gamma_{G \wedge}^{\nu \wedge}\right) \rightarrow \pi_{1}\left(\Gamma_{G}^{V}\right)$ is trivial,

(ii) $H_{1}\left(\Gamma_{G}^{V}\right)=H_{1}\left(\Gamma_{G \wedge}^{V \wedge}\right)=0$,

then every local extension over $V$ is enlargeable over $\hat{G}$ from $\hat{V}$ if and only if $\omega_{2}: H_{2}\left(\Gamma_{G \wedge}^{\nu \wedge}\right) \rightarrow H_{2}\left(\Gamma_{G}^{V}\right)$ is trivial.

This result has been established by P. A. Smith who took instead of (i), (ii) the somewhat stronger assumption that $\hat{G}$ and $G$ are $\hat{V}$ and $V$-monodrome (see [14, (50.7), p. 423]). Let us note that if $\hat{G}$ is $\hat{V}$-monodrome, then enlargeability from $\hat{V}$ over $\hat{G}$ is equivalent to just enlargeability from $\hat{V}$ (see $\S 2$, p. 294).

We conclude this section by discussing the enlargeability of a local extension $\mathfrak{L}$ in which $N$ is abelian. We shall show that in this case it may be possible to replace the second obstruction of $\mathfrak{L}$ over $G$ by a 2-dimensional cohomology class.

Suppose that $G$ is a group containing $V$ and generated by $V$ such that the kernel of $\mathfrak{E}$ is enlargeable over $G$, say to $\langle G, N, \Theta\rangle$. We have $C=N$ which shows that there exists a multiplicator $\langle\alpha, 1\rangle$ for $\langle G, N, \Theta\rangle$. Let $\mathscr{S}_{0}$ be the global extension defined by $\langle\alpha, 1\rangle$ and let $\mathfrak{L}_{0}$ be the subextension of $\mathscr{S}_{0}$ over $V$. The kernel of $\mathscr{S}_{0}$ being $\langle G, N, \Theta\rangle$ (by (b) in $\S 7$, p. 304), it is clear that $\mathfrak{L}_{0}, \mathfrak{L}$ have the same kernel. Hence $d\left(\mathfrak{Q}, \mathfrak{\Omega}_{0}\right)$ is defined. We denote by $\bar{\beta}^{2}: H^{2}(V, N) \rightarrow H^{2}\left(K_{G}^{V}, N\right)$ the morphism induced by $\operatorname{Hom}_{G}\left(K_{G}^{V}, N\right) \subset \operatorname{Hom}\left(K_{G}^{V}, N\right)$ where the $G$-structure of $N$ is given by $\alpha: G \rightarrow A(N)$.

LEMMA. If further to the above assumptions, $H_{1}\left(\Gamma_{G}^{V}\right)=0$, then $\mathfrak{L}$ is enlargeable over $G$ iff the element

vanishes.

$$
\tilde{\beta}^{2}\left(d\left(\mathfrak{L}, \mathfrak{\Omega}_{0}\right)\right) \in H^{2}\left(K_{G}^{V}, N\right)
$$

Proof. Since $\mathfrak{L}_{0}$ is enlargeable to $\mathfrak{S}_{0}$, the theorem in $\S 8$ (p. 305) implies

$$
\mathrm{Obs}_{\mathfrak{\Omega}_{0}}\langle G, N, \Theta\rangle=0 \text {. }
$$

Thus, by Lemma 3 of $\S 9$ (p. 311$), \mathfrak{L}$ is enlargeable over $G$ iff $d\left(\mathfrak{L}, \mathfrak{L}_{0}\right) \in \operatorname{Ker} \partial_{E}^{\text {ea }}$. It remains to observe now that we have a diagram

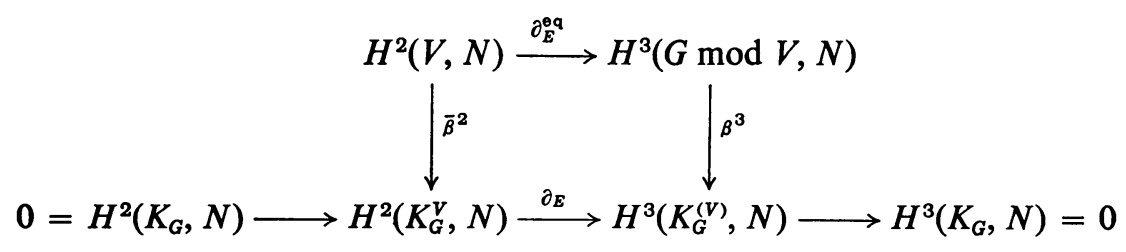

where $\beta^{3}$ is an isomorphism, by Lemma 1 in $\S 6$ (p. 300), whence $\operatorname{Ker} \partial_{E}^{\text {eaq }}=\operatorname{Ker} \bar{\beta}^{2}$. 
The above obstruction $\bar{\beta}^{2}\left(d\left(\mathfrak{L}, \mathfrak{L}_{0}\right)\right)$ has been used in [6, Proposition 1.1, p. 17] for the construction of a nonenlargeable normed Lie algebra (see $\S 12$ below).

11. Extensions over topological local groups. Let $G$ be a topological group and let $V$ be a symmetric neighborhood of the identity. We shall deduce from Corollary 2 in $\$ 10$ the

THEOREM. Assume that the topological space $G$ is 1-connected and such that its second singular homology $H_{2}^{\mathrm{top}}(G)$ vanishes. Let $\hat{V}, Q$ be open symmetric neighborhoods of the identity satisfying

(i) $\hat{V} \hat{V} \subset Q, Q Q \subset V$,

(ii) $\hat{V}$ is connected,

(iii) every closed curve in $\hat{V} \hat{V}$ is contractible in $Q$.

Then every local extension over $V$ is enlargeable over $G$ from $\hat{V}$.

Related theorems are in [13 (without proof) and in ([5, Theorem 7.1, p. 399]). Let us show first that the 1-connectedness of $G$ and (ii) imply that $G$ is $\hat{V}$ monodrome. Let $\hat{G}$ be the $\hat{V}$-monodrome group. Then the topology on $\hat{V}$ can be extended to $\hat{G}$, so that $\hat{G}$ becomes a topological group [2, Theorem 2.7.1, p. 50], moreover $\hat{G}$ will be connected because it is generated by the connected $\hat{V}$. The inclusion $\hat{V} \subset G$ can now be enlarged to a morphism $\hat{G} \rightarrow G$.

On the other hand, $G$ being 1-connected and locally isomorphic to $\hat{G}$, is the simply connected covering group of $\hat{G}$, so that we have a covering morphism $G \rightarrow \hat{G}$ which on $\hat{V}$ is the identity. The two morphisms between $\hat{G}, G$ are inverse to each other (since their restrictions to $\hat{V}$ are), hence $G$ is isomorphic to $G$, and $G$ is $\hat{V}$-monodrome.

Let $G, V, Q, \hat{V}$ satisfy the assumptions of the theorem. Then conditions (i), (ii) of Corollary 2 in $\S 10$ (p. 313 ) are satisfied with $\hat{G}=G$. To prove the theorem, it remains to show that

$$
\omega_{2}: H_{2}\left(\Gamma_{G \wedge}^{V \wedge}\right) \rightarrow H_{2}\left(\Gamma_{G}^{V}\right)
$$

is trivial. We denote by $S=\left\{S_{n}, \partial\right\}_{n \geqq 0}$ the complex of singular chains of the topological space $G$. Let further $S^{Q}=\left\{S_{n}^{Q}, \partial\right\}_{n \geqq 0}$ be the subcomplex of $S$ generated (as abelian group) by singular simplexes of the form

$$
s: J_{n} \rightarrow G ; n=0,1,2, \ldots ; \text { where } s\left(J_{n}\right) \subset x Q \text { for some } x \in G,
$$

( $J_{n}$ is the standard simplex $\left\{\left\langle t_{0}, \ldots, t_{n}\right\rangle \in R^{n+1} \mid \sum t_{i}=1, t_{i} \geqq 0\right\}$ ). Since $\{x Q\}_{x \in G}$ is an open covering of $G$, if follows from known results [4, Chapter VII, Theorem 8.2 , p. 197] that the inclusion $S^{Q} \subset S$ is a chain equivalence. Hence the singular homology of $G$ equals the homology of the chain complex $S^{Q}$, so we conclude that

$$
H_{2}\left(S^{Q}\right)=0 .
$$


Let us define a morphism $f: S^{Q} \rightarrow \mathfrak{G}\left(\Gamma_{G}^{V}\right)$ by defining in each dimension $f_{n}: S_{n}^{Q} \rightarrow \mathbb{E}_{n}\left(\Gamma_{G}^{V}\right)$ as follows: If

$$
s: J_{n} \rightarrow x Q
$$

belongs to $S_{n}^{Q}$, then the image of this simplex under $f_{n}$ is

$$
\begin{gathered}
\left\langle g_{0}, g_{1}, \ldots, g_{n}\right\rangle \text {, where } g_{i}=s\left\langle t_{0}, \ldots, t_{i}, \ldots, t_{n}\right\rangle \\
\text { with } t_{i}=1 \text { and } t_{j}=0 \text { for } j \neq i,
\end{gathered}
$$

$\left(g_{i}\right.$ is the " $i$ th vertex" of $\left.s: J_{n} \rightarrow x Q\right)$. The map is well defined since

$$
g_{i}^{-1} g_{\jmath} \in Q x^{-1} x Q \subset V .
$$

It is not hard to see that $f: S^{Q} \rightarrow \mathfrak{C}\left(\Gamma_{G}^{V}\right)$ is a chain map.

We assert that it will suffice now to prove the existence of two morphisms $h_{i} \in \operatorname{Hom}\left(\mathbb{E}_{i}\left(\Gamma_{G \wedge}^{V \wedge}\right), S_{i}^{Q}\right) ; i=1,2$, such that the upper square of the following diagram

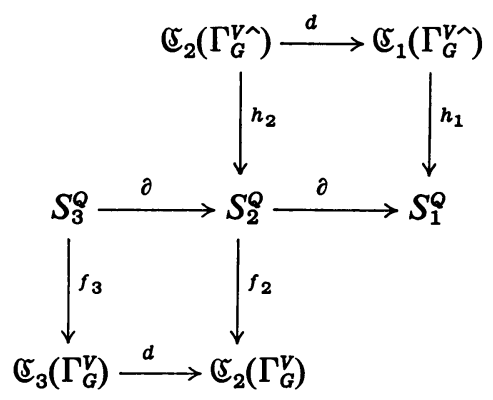

commutes, and $f_{2} \circ h_{2}: \mho_{2}\left(\Gamma_{G}^{V \wedge}\right) \rightarrow \mathbb{\mho}_{2}\left(\Gamma_{G}^{\nu}\right)$ is induced by the inclusion $\Gamma_{G}^{V} \subset \Gamma_{G}^{\nu \wedge}$. Indeed, it follows then from the exactness at $S_{2}^{\varrho}(11.1)$ that every cycle in $\mathcal{C}_{2}\left(\Gamma_{G}^{\nu \wedge}\right)$ is a boundary in $\mathfrak{E}_{2}\left(\Gamma_{G}^{V}\right)$, thus $\omega_{2}: H_{2}\left(\Gamma_{G}^{V \wedge}\right) \rightarrow H_{2}\left(\Gamma_{G}^{V}\right)$ vanishes.

The morphism $h_{1}$. A typical free generator of $\mathfrak{C}_{1}\left(\Gamma_{G}^{\left.\gamma^{\wedge}\right)}\right.$ is of the form $\left\langle g_{0}, g_{1}\right\rangle$ where $g_{0}^{-1} g_{1} \in \hat{V}$. Thus $g_{1} \in g_{0} \hat{V}$, and since $g_{0} \hat{V}$ is connected, there is a singular 1-simplex

$$
s_{1}: J_{1} \rightarrow g_{0} \hat{V}
$$

such that $s_{1}\langle 1,0\rangle=g_{0}, s_{1}\langle 0,1\rangle=g_{1}$. We denote this 1 -simplex by $h_{1}\left\langle g_{0}, g_{1}\right\rangle$. From $\hat{V} \subset Q$ follows $h_{1}\left\langle g_{0}, g_{1}\right\rangle \in S_{1}^{Q}$. We extend now $h_{1}$ by linearity to $\mathfrak{C}_{1}\left(\Gamma_{G}^{V \wedge}\right) \rightarrow S_{1}^{Q}$.

The morphism $h_{2}$. Let $\left\langle g_{0}, g_{1}, g_{2}\right\rangle$ be a free generator of $\mathfrak{C}_{2}\left(\Gamma_{G}^{V \wedge}\right)$. Then $g_{i}^{-1} g_{j} \in \hat{V}$, hence $g_{i} \in g_{0} \hat{V}, g_{2} \in g_{1} V$ and $g_{1} \hat{V} \subset g_{0} \hat{V} \hat{V}$, so that we have the above defined singular simplexes

$$
h_{1}\left\langle g_{0}, g_{1}\right\rangle, h_{1}\left\langle g_{0}, g_{2}\right\rangle, h_{1}\left\langle g_{1}, g_{2}\right\rangle: J_{1} \rightarrow g_{0} \hat{V} \hat{V} .
$$

These form a "curvilinear triangle" with vertices $g_{0}, g_{1}, g_{2}$ contained entirely in 
$g_{0} \hat{V} \hat{V}$. Since every closed curve in $\hat{V} \hat{V}$ is by assumption contractible in $Q$, there exists a singular 2-simplex

$$
s_{2}: J_{2} \rightarrow g_{0} Q
$$

belonging to $S_{2}^{Q}$, such that

$$
\partial s_{2}=h_{1}\left\langle g_{0}, g_{1}\right\rangle+h_{1}\left\langle g_{1}, g_{2}\right\rangle-h_{1}\left\langle g_{0}, g_{2}\right\rangle=h_{1}\left(d\left\langle g_{0}, g_{1}, g_{2}\right\rangle\right) .
$$

We define $h_{2}\left\langle g_{0}, g_{1}, g_{2}\right\rangle=s_{2}$ and we extend $h_{2}$ to $\mathfrak{C}_{2}\left(\Gamma_{G}^{V \wedge}\right) \rightarrow S_{2}^{Q}$ by linearity. Then (11.3) yields $\partial h_{2}=h_{1} d$, that is, the upper square of (11.2) commutes. It is clear that "the vertices" of $h_{2}\left\langle g_{0}, g_{1}, g_{2}\right\rangle$ are $g_{0}, g_{1}, g_{2}$, hence $f_{2} \circ h_{2}$ is induced by the inclusion $\Gamma_{G}^{V \wedge} \subset \Gamma_{G}^{V}$.

12. Enlargeable normed Lie algebras. A Lie algebra $X$ will be called normed if $X$ is also a Banach space and the Lie product $[x, y]$ is a continuous function $X \times X \rightarrow X$. If $Y$ is an ideal of $X$ (all ideals are assumed to be closed subspaces), then $X / Y$, with the quotient norm $|x+y|=\inf \{|x+y||y \in Y|\}$, is again a normed Lie algebra. Given a normed Lie algebra $X$, we denote by $L_{r}(X)$ the open ball in $X$ with centre 0 and radius $r$. If we choose $r$ sufficiently small and say that the product $x y$ of $x, y \in L_{r}(X)$ exists iff the Campbell-Hausdorff series

$$
x+y+\frac{1}{2}[x, y]+\frac{1}{12}[y,[y, x]]+\cdots
$$

converges to an element $z$ in $L_{r}(X)$, and if we put $x y=z$, then $L_{r}(X)$ becomes a local group (see [15, p. 207, 212] for references). We shall call $X$ enlargeable if there exists an $r>0$ such that the local group $L_{r}(X)$ is enlargeable. If $L_{r}(X)$ is contained in a group $G$, we can extend the topology in $L_{r}(X)$ to a connected topology of $G$ [2, Theorem 2.7 .1$, p. 50], and if $G$ is not 1-connected, let us consider its 1-connected covering group; the latter will still contain the 1-connected subspace $L_{r}(X)$. Moreover, this covering group will be $L_{s}(X)$-monodrome for every $s \leqq r$ (see first part of the proof in $\S 11$, p. 315), and therefore it depends only on $X$. This unique (if it exists) 1-connected group containing some $L_{r}(X)$ will be denoted by $G(X)$.

Nonenlargeable normed Lie algebras exist, an example is given in [6]. We shall deduce from the result of the previous section the

THEOREM. If a normed Lie algebra $X$ contains an ideal $Y$ such that

(i) $Y$ is enlargeable,

(ii) $X / Y$ is enlargeable,

(iii) $H_{2}^{\text {top }}(G(X / Y))=0$, then $X$ is enlargeable.

COROLlary 1. If $X$ contains an enlargeable ideal $Y$ such that $X / Y$ is abelian or finite dimensional, then $X$ is enlargeable. 
Indeed, it follows from (12.1) that an abelian Lie algebra $X$ is always enlargeable and $G(X)=X$. The enlargeability of finite dimensional Lie algebras is well known [8, Chapter XII, Theorem 1.1], moreover a 1-connected Lie group is also 2-connected (see [1]) whence by the Hurewicz theorem [10, Corollary 9.2, p. 57] its second singular homology vanishes.

COROllary 2. If the centre of $X$ is of finite codimension, then $X$ is enlargeable.

Indeed, $X$ /centre $X$ is always enlargeable (this can be shown similarly as for finite dimensional $X($ see $[12, \S 54, \mathrm{~F}, \mathrm{p} .175]))$. Let us note that Corollary 2 yields a new proof of the enlargeability of finite dimensional Lie algebras based on the vanishing of the second homology of a 1-connected Lie group.

Proof of the theorem. The exact sequence $0 \rightarrow Y \subset X \rightarrow_{p} X / Y \rightarrow 0$, when restricted to balls of sufficiently small radius $r$, yields an exact sequence of local groups

$$
\sum_{r}: 1 \longrightarrow L_{r}(Y) \subset L_{r}(X) \underset{p}{\longrightarrow} L_{r}(X / Y) \longrightarrow 1
$$

Taking $r$ small enough, we can assume that $L_{r}(Y) \subset N=N(Y), L_{r}(X) \subset G=G(X / Y)$. Let $s<\bar{s}<r$ be such that the product of any 3 elements of $L_{\bar{s}}(X)$ exists in $L_{r}(X)$ and the product of any 14 elements of $L_{s}(X)$ exists in $L_{s}(X)$ (these products should exist independently on the way of inserting the brackets; the existence of $s, \bar{s}$ follows from the continuity of multiplication in $L_{r}(X)$ ). We shall define an extension $\mathfrak{Q}$ such that there is a commutative diagram

$$
\begin{aligned}
& \sum_{s}: 1 \longrightarrow L_{s}(Y) \subset L_{s}(X) \underset{p}{\longrightarrow} L_{s}(X / Y) \longrightarrow 1 \\
& \mathfrak{L}: 1 \longrightarrow N \subset U \longrightarrow
\end{aligned}
$$

Indeed, if we have such a diagram, we can take balls $Q=L_{q}(X / Y), \hat{V}=L_{t}(X / Y)$ such that the assumptions (i), (ii), (iii) of the Theorem in $\S 11,($ p. 315) are satisfied. By that theorem, $\mathfrak{Q}$ is enlargeable from $L_{t}(X / Y)$, hence $\phi^{-1} L_{t}(X / Y)$ is enlargeable. But $L_{t}(X) \subset p^{-1} L_{t}(X / Y) \subset \phi^{-1} L_{t}(X / Y)$. Hence $X$ is enlargeable.

We shall associate now with $\sum_{s}$ and a given $p$-selector a multiplicator $\langle\alpha, h\rangle$ from $V$ to $N$, and $\mathfrak{Q}$ will be the extension defined by $\langle\alpha, h\rangle$.

Let us denote by $\left(L_{s}(X)\right)^{k}$ the subset $\left\{x_{1} \ldots x_{k} \mid x_{i} \in L_{s}(X)\right\}$ of $L_{r}(X)$; for $k \leqq 7$ this is evidently a local group. For each $x$ in $\left(L_{s}(X)\right)^{3}$ we consider the map $L_{s}(Y)$ $\rightarrow L_{r}(Y) \subset N$ which sends $y \in L_{s}(Y)$ to $x y x^{-1} \in\left(L_{s}(X)\right)^{7} \cap L_{r}(Y)$. It is readily checked that this map is a morphism, and as $N$ is $L_{s}(Y)$-monodrome (see beginning of the proof in $\S 11)$, we can uniquely extend it to a morphism $\iota_{N}(x): N \rightarrow N$. If, for given $x \in\left(L_{s}(X)\right)^{3}, W \subset N$ is a neighborhood of the identity satisfying $x W x^{-1} \subset L_{s}(Y)$, then $\iota_{N}\left(x^{-1}\right) \iota_{N}(x): N \rightarrow N$ is on $W$ equal to the identity, and as $W$ generates $N$, we conclude that $\iota_{N}(x)$ has a right inverse, hence (interchanging the 
roles of $x$ and $\left.x^{-1}\right) \iota_{N}(x) \in A(N)$. The map $\iota_{N}:\left(L_{s}(X)\right)^{3} \rightarrow A(N)$ is a morphism: it is easily verified that if $x, y, y x \in\left(L_{s}(X)\right)^{3}$, then the morphisms $\iota_{N}(y) \iota_{N}(x), \iota_{N}(y x)$ are identical on the above $W$, and hence on $N$. We have by a similar argument that if $x \in\left(L_{s}(X)\right)^{3} \cap L_{r}(Y) \subset N$, then $\iota_{N}(x)=\iota(x)$ is the inner automorphism of $N$ by $x$.

Let $\psi: V=L_{s}(X / Y) \rightarrow L_{s}(X)$ be a $p$-selector. Then for $g_{1}, g_{2}, g_{1} g_{2} \in V$, we have an element

$$
h\left(g_{1}, g_{2}\right)=\psi\left(g_{1}\right) \psi\left(g_{2}\right)\left(\psi\left(g_{1} g_{2}\right)\right)^{-1} \in\left(L_{s}(X)\right)^{3} \cap L_{r}(Y) \subset N .
$$

Let $\alpha: V \rightarrow A(N)$ be defined by $\alpha=\iota_{N} \circ \psi$. Applying $\iota_{N}$ to the above equality, we get $\iota\left(h\left(g_{1}, g_{2}\right)\right)=\alpha\left(g_{1}\right) \alpha\left(g_{2}\right)\left(\alpha\left(g_{1} g_{2}\right)\right)^{-1}$. Hence $\langle\alpha, h\rangle$ is a premultiplicator from $V$ to $N$; to show that it is a multiplicator we need the

LemMA. If $y \in L_{\hat{S}}(Y), z \in\left(L_{s}(X)\right)^{3}$, then $z y z^{-1}={ }^{\iota_{N}(z)} y$.

For the proof we use the following fact valid for any local group $\Omega$ : If $z, y_{1}, \ldots$, $y_{n} \in \Omega$ are such that the product $y_{1} y_{2} \cdots y_{n}$ exists (with every inserting of brackets), and for each $i=1, \ldots, n$ the products $z y_{i}, y_{i} z^{-1}, z y_{i} z^{-1}, z y_{1} \cdots y_{i}, y_{1} \cdots y_{i} z^{-1}$ and $z y_{1} \cdots y_{i} z^{-1}$ exist, then

$$
\left(z y_{1} \cdots y_{i} z^{-1}\right)\left(z y_{i+1} z^{-1}\right)=z y_{1} \cdots y_{i+1} z^{-1} \text { for } i=1, \ldots, n-1 .
$$

(This is easily shown by a multiple application of the associativity laws.)

To prove the lemma, we note first that if for real $\alpha, \beta$ we have $\alpha y, \beta y,(\alpha+\beta) y$ $\in L_{r}(Y)$, then $(\alpha y)(\beta y)=(\alpha+\beta) y$, by (12.1). So for $\gamma \in L_{\hat{s}}(Y)$, we have $y=y_{1} y_{2} \cdots y_{n}$ with $y_{i}=(1 / n) y, y_{1} \cdots y_{i} \in L_{\bar{s}}(Y)$ for $i=1, \ldots, n$ and $y_{1} \cdots y_{n}$ exists with arbitrary brackets. Taking $n$ large enough, we shall have $y_{i} \in L_{s}(Y)$, whence $z y_{i} z^{-1}={ }^{t_{N}(z)} y_{i}$ by the definition of $\iota_{N}$. The assumptions which led to (12.3) are satisfied (with $\Omega$ $\left.=L_{r}(X)\right)$, whence

$$
\left(z y_{1} \cdots y_{i} z^{-1}\right)\left({ }^{{ }{ }^{(}(z)} y_{i}\right)=z y_{1} \cdots y_{i+1} z^{-1} \text { for } i=1, \ldots, n-1 .
$$

These equations are in $N$, so it follows that

$$
\left({ }^{{ }(z)} y_{1}\right)\left({ }^{{ }}(z) y_{2}\right) \cdots\left({ }^{{ } N}(z) y_{n}\right)=z y_{1} \cdots y_{n} z^{-1} .
$$

This proves the lemma.

It is easily seen that every $x \in L_{s}(X)$ can be uniquely written as $x=y \psi(g)$ where $g=p(x)$ and $y \in\left(L_{s}(X)\right)^{2} \cap L_{r}(Y) \subset N$. Moreover, if $g_{1}, g_{2}, g_{1} g_{2} \in V$,

$$
\begin{aligned}
x_{1} x_{2}=y_{1} \psi\left(g_{1}\right) y_{2} \psi\left(g_{2}\right) & =y_{1}{ }^{\alpha\left(g_{1}\right)} y_{2} \psi\left(g_{1}\right) \psi\left(g_{2}\right) \\
& =y_{1}{ }^{\alpha\left(g_{1}\right)} y_{2} h\left(g_{1}, g_{2}\right) \psi\left(g_{1} g_{2}\right),
\end{aligned}
$$

for we have $\psi\left(g_{1}\right) y_{2}\left(\psi\left(g_{1}\right)\right)^{-1}=\alpha\left(g_{1}\right) y_{2}$ by the above lemma taken with $z=\psi\left(g_{1}\right)$, $y=y_{2}$. Now, if $g_{1}, g_{2}, g_{3}, g_{1} g_{2}, g_{2} g_{3}, g_{1} g_{2} g_{3} \in V$, then we can calculate $\psi\left(g_{1}\right) \psi\left(g_{2}\right)$ $\cdot \psi\left(g_{3}\right)$ in two different ways with the aid of $\psi\left(g_{i}\right) \psi\left(g_{j}\right)=h\left(g_{i}, g_{j}\right) \psi\left(g_{i} g_{j}\right)$, which is a consequence of (12.4), and using $\psi\left(g_{1}\right) h\left(g_{2}, g_{3}\right)\left(\psi\left(g_{1}\right)\right)^{-1}={ }^{\alpha\left(g_{1}\right)} h\left(g_{2}, g_{3}\right)$, which is a consequence of the lemma. In this way we arrive at $f_{\alpha, h}=1$. Let $\mathfrak{L}$ be the extension 
defined by $\langle\alpha, h\rangle$. Then the identification of $y \psi(g) \in L_{s}(X)$ with $\langle y, g\rangle \in U$ gives, by (12.4), an inclusion of local groups $L_{s}(X) \subset U$ commuting with $p$ and $\phi$. Thus the diagram (12.2) commutes.

\section{REFERENCES}

1. W. Browder, Torsion in H-spaces, Ann. of Math. 74 (1961), 24-51.

2. P. M. Cohn, Lie groups, Cambridge Tracts in Mathematics and Math. Phys. 46 (1961).

3. S. Eilenberg and S. MacLane, Cohomology theory in abstract groups, Ann. of Math. 48 (1947), 51-78 and 326-341.

4. S. Eilenberg and N. Steenrod, Foundations of algebraic topology, Princeton Univ. Press, Princeton, N. J., 1946.

5. W. T. van Est, Local and global groups, Indag. Math. 24 (1962), 391-425.

6. W. T. van Est and Th. J. Korthagen, Non-enlargible Lie algebras, Indag. Math. 26 (1964), 15-31.

7. R. Godement, Topologie algébrique et théorie des faisceaux, Actualités Sci. Ind. No. 1252, Hermann, Paris, 1964.

8. G. Hochschild, The structure of Lie groups, Holden-Day, San Francisco, Calif., 1965.

9. G. Hochschild and J. P. Serre, Cohomology of group extensions, Trans. Amer. Math. Soc. 74 (1953), 110-134.

10. Shih-chen Hu, Homotopy theory, Academic Press, New York, 1959.

11. S. MacLane, Homology theory, Springer, Berlin, 1963.

12. L. S. Pontrjagin, Topologische Gruppen, Teubner, Leipzig, 1958.

13. P. A. Smith, Some topological notions connected with a set of generators, Proc. Int. Congr. of Mathematicians, Cambridge, Mass., 1950, Vol. II, Amer. Math. Soc., 1952, pp. 436-441.

14. - The complex of a group relative to a set of generators, Ann. of Math. 54 (1951), 371-424.

15. S. Świerczkowski, Embedding theorems for local analytic groups, Acta Math. 114 (1965), 207-235.

16. - Homology and cohomology of abstract local groups (to appear).

17. H. Zassenhaus, Lehrbuch der Gruppentheorie, Teubner, Leipzig, 1937.

THE INSTITUTE FOR ADVANCED STUDY,

Princeton, New Jersey

THE UNIVERSITY OF SUSSEX,

BRIGHTON, ENGLAND 